\title{
On the expected discounted dividends in the Cramér-Lundberg risk model with more frequent ruin monitoring than dividend decisions
}

\author{
Michael C.H. Choi* and Eric C.K. Cheung ${ }^{\dagger}$
}

August 26, 2014

\begin{abstract}
In this paper, we further extend the insurance risk model in Albrecher et al. (2011b), who proposed to only intervene in the compound Poisson risk process at the discrete time points $\left\{L_{k}\right\}_{k=0}^{\infty}$ where the event of ruin is checked and dividend decisions are made. In practice, an insurance company typically balances its books (and monitors its solvency) more frequently than deciding on dividend payments. This motivates us to propose a generalization in which ruin is monitored at $\left\{L_{k}\right\}_{k=0}^{\infty}$ whereas dividend decisions are only made at $\left\{L_{j k}\right\}_{k=0}^{\infty}$ for some positive integer $j$. Assuming that the intervals between the time points $\left\{L_{k}\right\}_{k=0}^{\infty}$ are $\operatorname{Erlang}(n)$ distributed, the Erlangization technique (e.g. Asmussen et al. (2002)) allows us to model the more realistic situation with the books balanced e.g. monthly and dividend decisions made e.g. quarterly or semi-annually. Under a dividend barrier strategy with the above randomized interventions, we derive the expected discounted dividends paid until ruin. Numerical examples about dividend maximization with respect to the barrier $b$ and/or the value of $j$ are given.
\end{abstract}

Keywords: Compound Poisson risk model; Barrier strategy; Dividend decisions; Randomized observations; Erlangization.

\section{Introduction and preliminaries}

In this paper, we model the insurer's surplus (in the absence of dividends) using the classical compound Poisson (or Cramér-Lundberg) risk process $\{U(t)\}_{t \geq 0}$, where

$$
U(t)=x+c t-\sum_{i=1}^{N(t)} Y_{i}, \quad t \geq 0 .
$$

Here $x=U(0) \geq 0$ is the initial surplus level, $c>0$ is the constant premium rate received by the insurer, $\{N(t)\}_{t \geq 0}$ is a Poisson process with rate $\lambda>0$, and $\left\{Y_{i}\right\}_{i=1}^{\infty}$ are the claim severities which form a sequence of independent and identically distributed (i.i.d.) positive random variables independent of $\{N(t)\}_{t \geq 0}$. It is assumed that each $Y_{i}$ has the same distribution as a generic continuous random variable $Y$. For later use, we define $f_{Y}(\cdot)$ and $\widetilde{f}_{Y}(\cdot)$ respectively to be the density and the Laplace transform of $Y$. Traditionally, ruin is said to occur when the process $\{U(t)\}_{t \geq 0}$ drops below zero for the first time.

\footnotetext{
${ }^{*}$ School of Operations Research and Information Engineering, Cornell University, Ithaca, New York.

${ }^{\dagger}$ Department of Statistics and Actuarial Science, University of Hong Kong, Pokfulam, Hong Kong.
} 
It is well known (e.g. Asmussen and Albrecher (2010, Proposition 1.2)) that if the positive loading condition $c>\lambda \mathbb{E}[Y]$ holds, then the insurer's surplus will grow indefinitely in the long run. This led de Finetti (1957) to propose that the insurance company should redistribute part of the surplus to its shareholders as dividends. Comprehensive reviews of various dividend strategies and optimality results can be found in Albrecher and Thonhauser (2009) and Avanzi (2009). In the literature, the dividend strategy that is studied the most is the (horizontal) barrier strategy, which assumes that dividends are paid to the shareholders at rate $c$ immediately when the surplus reaches a fixed barrier $b \geq 0$ until the next claim occurs, and no further dividends are payable after the modified process is ruined. See e.g. Gerber (1979), Gerber and Shiu (1998, Section 7), Lin et al. (2003), Dickson and Waters (2004) and Gerber et al. (2006). The barrier strategy is known to be optimal in maximizing the expected discounted dividends until ruin when $f_{Y}(\cdot)$ is completely monotone (e.g. Loeffen (2008, Theorem 3$)$ ).

Noting that the implementation of the traditional barrier strategy in the model (1.1) would result in dividends that are payable in the form of continuous streams, Albrecher et al. (2011b, 2013) proposed to only 'look' at the process at the observation times $\left\{L_{k}\right\}_{k=0}^{\infty}$ with $L_{0}=0$. At these time points, if the surplus level is above the barrier $b$ then the excess is paid out as dividend to the shareholders; but if the surplus is observed to be below zero then ruin is declared (and dividend payments cease after ruin). In this way, the more realistic situation where the insurer balances its books periodically and at the same time the board of the company decides whether to pay (lump sum) dividends can be modelled. Defining $T_{k}=L_{k}-L_{k-1}$ for $k=1,2, \ldots$, Albrecher et al. (2011b, 2013) assumed that the inter-observation times $\left\{T_{k}\right\}_{k=1}^{\infty}$ form an i.i.d. sequence with the same distribution as $T$ and is independent of $\{N(t)\}_{t \geq 0}$ and $\left\{Y_{i}\right\}_{i=1}^{\infty}$. In particular, motivated by the Erlangization technique commonly used in finite-time ruin problems (e.g. Asmussen et al. (2002), Stanford et al. (2005, 2011), and Ramaswami et al. (2008)), it is assumed that $T$ follows an $\operatorname{Erlang}(n)$ distribution with density

$$
f_{T}(t)=\frac{\gamma^{n} t^{n-1} e^{-\gamma t}}{(n-1) !}, \quad t>0
$$

and Laplace transform $\widetilde{f}_{T}(s)=\int_{0}^{\infty} e^{-s t} f_{T}(t) d t=[\gamma /(\gamma+s)]^{n}$, where $\gamma>0$. In fact, if the mean $\mathbb{E}[T]=n / \gamma$ is fixed to be $h$ and the value of $n$ is increased (along with $\gamma$ ), then $T$ converges in distribution to a point mass at $h$, approximating the case of deterministic inter-observation times (e.g. monthly balancing of books). Interested readers are also referred to e.g. Carr (1998) and Kyprianou and Pistorius (2003) for the use of Erlangization in option pricing.

Since the idea of randomized observations was proposed, a number of related research problems have been analyzed. Some optimality results concerning dividend maximization when $\left\{L_{k}\right\}_{k=0}^{\infty}$ are more generally the arrival epochs of a renewal process were derived by Albrecher et al. (2011a). Discussions of exponential inter-observation times in relation to the (Gamma-)Omega risk model can also be found in Albrecher et al. (2011c, Section 9), Gerber et al. (2012, Section 3), and Albrecher and Lautscham (2013, Section 2.1.1). In addition, Albrecher and Ivanovs (2013) and Albrecher et al. (2014) studied the cases of a Poissonian observer (i.e. the observation time points $\left\{L_{k}\right\}_{k=0}^{\infty}$ are the arrival times of a Poisson process that may be non-homogeneous) in a Markovian environment and a Lévy risk process respectively; whereas Chen et al. (2014) considered a threshold dividend strategy under exponential inter-observation times. Another development of randomized observations was suggested by Avanzi et al. (2013), who argued that only dividend announcements are made periodically but the financial position of a company should be monitored continuously. In other words, ruin is declared immediately once the company's surplus becomes negative in their modifications. Although the original work by Avanzi et al. (2013) is concerned with the dual risk model (see also Avanzi et al. (2014) for optimality results), insurance risk 
processes with periodic dividend decisions and continuous monitoring of solvency have also been studied by Zhang (2014) and Zhang and Cheung (2014a,b).

Inspired by Avanzi et al. (2014)'s comments, we aim at further refining Albrecher et al. (2011b)'s model in this paper as follows. In practice, the solvency of the insurer is monitored more frequently than dividend decisions are made such that an insurer balances its books (and monitors its solvency) on e.g. a monthly basis while dividends are announced e.g. quarterly or semi-annually. Therefore, we propose a generalized model in which ruin is monitored at the observation times $\left\{L_{k}\right\}_{k=0}^{\infty}$ whereas dividend decisions are only made at a subset of these times, namely $\left\{L_{j k}\right\}_{k=0}^{\infty}$ for some positive integer $j$. With Erlangization in mind, it is still assumed that each $T_{k}=L_{k}-L_{k-1}$ (for $k=1,2, \ldots$ ) follows the Erlang $(n)$ density (1.2), and a barrier-type strategy is applied. To define the modified surplus process $\left\{U_{b}(t)\right\}_{t \geq 0}$ under the above descriptions, we first introduce the auxiliary process $\left\{U_{b, k}(t)\right\}_{t \geq 0}$ which can be described recursively via

$$
U_{b, k}(t)=\left\{\begin{array}{lll}
U(t), & k=1 ; & t \geq 0 . \\
\min \left(U_{b, k-1}\left(L_{j(k-1)}\right), b\right)+U(t)-U\left(L_{j(k-1)}\right), & k=2,3, \ldots ; & t \geq L_{j(k-1)} .
\end{array}\right.
$$

Then, for $k=1,2, \ldots$, one has

$$
U_{b}(t)=U_{b, k}(t), \quad L_{j(k-1)} \leq t<L_{j k} .
$$

Without loss of generality, it is assumed that the initial surplus level $U_{b}(0)=U_{b, 1}(0)=U(0)=x$ is such that $0 \leq x \leq b$. Defining $k_{b}=\inf \left\{k \geq 1: U_{b}\left(L_{k}\right)<0\right\}$ to be the number of observation periods before ruin, the time of ruin of the modified surplus process $\left\{U_{b}(t)\right\}_{t \geq 0}$ is given by $\tau_{b}=L_{k_{b}}$. Clearly, when $j=1$ our model reduces to the one by Albrecher et al. (2011b). In addition, the Erlangization technique allows us to model the realistic situation of monthly balancing of books along with quarterly or semi-annual dividend decisions by letting $j=3$ or $j=6$. Figure 1 depicts a sample path of $\left\{U_{b}(t)\right\}_{t \geq 0}$ when $j=2$.

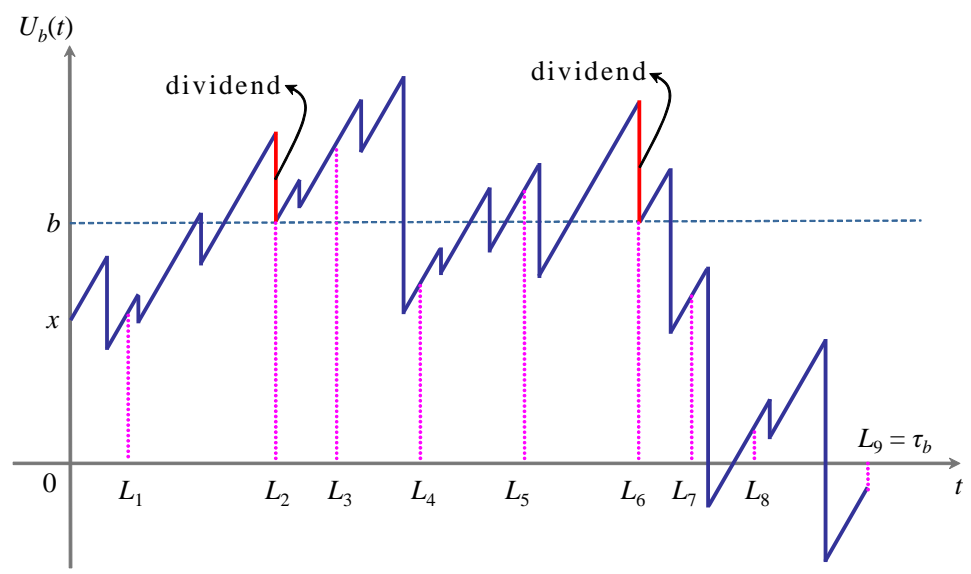

Figure 1: A sample path of $\left\{U_{b}(t)\right\}_{t \geq 0}$ when $j=2$

The key quantity of interest in this paper is the expected discounted dividends paid until ruin defined by

$$
V(x ; b)=\mathbb{E}\left[\sum_{k=1}^{\infty} e^{-\delta L_{j k}}\left[U_{b}\left(L_{j k}^{-}\right)-b\right]_{+} 1_{\left\{L_{j k}<\tau_{b}\right\}} \mid U_{b}(0)=x\right], \quad 0 \leq x \leq b,
$$


where $\delta>0$ is the force of interest, $a_{+}=\max (a, 0)$, and $1_{A}$ is the indicator function of the event $A$. While the traditional dividend maximization under a barrier strategy is performed with respect to the barrier level $b$ (e.g. Gerber et al. (2006)), in the present context one can also optimize with respect to $j$ (see Section 4). This could address the following type of question: given that the insurer balances its books monthly, is it better to pay dividend quarterly, semi-annually or annually? Our analysis of the dividend function $V(x ; b)$ in the upcoming sections relies heavily on the so-called discounted density of the increment of the barrier-free process $\{U(t)\}_{t \geq 0}$ between successive observation time points (see Albrecher et al. (2013, Section 3.2)). It is defined via the joint Laplace transform of $\left(T, \sum_{i=1}^{N(T)} Y_{i}-c T\right)$, namely

$$
\mathbb{E}\left[e^{-\delta T-s\left(\sum_{i=1}^{N(T)} Y_{i}-c T\right)}\right]=\left(\frac{\gamma}{\gamma+\delta-c s+\lambda\left[1-\widetilde{f}_{Y}(s)\right]}\right)^{n}=\int_{-\infty}^{\infty} e^{-s y} g_{\delta}(y) d y .
$$

The density $g_{\delta}(\cdot)$ can be further decomposed into

$$
g_{\delta}(y)=g_{\delta,-}(-y) 1_{\{y<0\}}+g_{\delta,+}(y) 1_{\{y>0\}}, \quad-\infty<y<\infty,
$$

where $g_{\delta,+}(\cdot)$ and $g_{\delta,-}(\cdot)$ represent the cases of net loss and net gain respectively between observations (prior to any dividend payments). These densities are known to take on tractable form when the claim severity $Y$ has rational Laplace transform and the inter-observation time $T$ follows $\operatorname{Erlang}(n)$ distribution (see $(3.2))$.

This paper is organized as follows. In Section 2, the assumption that both the claim severity $Y$ and the inter-observation time $T$ are exponentially distributed is made. We first start with the case $j=2$ in Section 2.1 which will give some insights to the more general case of $j \in \mathbb{N}$ considered in Section 2.2. The densities $g_{\delta,+}(\cdot)$ and $g_{\delta,-}(\cdot)$ will be used to derive integral equations satisfied by $V(x ; b)$, which can be solved explicitly. Although most of the ideas can be found in Section 2.2, we further study the general but tedious case where $Y$ has rational Laplace transform, $T$ is $\operatorname{Erlang}(n)$ and $j \in \mathbb{N}$ in Section 3 for the sake of completeness. Finally, Section 4 is concerned with various numerical illustrations which involve dividend maximization with respect to the barrier level $b$ and/or the value of $j$.

\section{Exponential claims and exponential inter-observation times}

In this entire section, it is assumed that $Y$ and $T$ follow exponential distribution with mean $1 / \nu$ and $1 / \gamma$ respectively. We shall first investigate the case $j=2$ and then turn to the more general case $j \in \mathbb{N}$.

\subsection{Model with $j=2$}

Under $j=2$, solvency is monitored at the observation times $\left\{L_{k}\right\}_{k=0}^{\infty}$ while dividend decisions are only made at $\left\{L_{2 k}\right\}_{k=0}^{\infty}$. With dividend decisions made at a subset of $\left\{L_{k}\right\}_{k=0}^{\infty}$, one needs to keep track of the number of inter-observation times remaining until the next dividend decision. To this end, for $0 \leq x \leq b$ we denote $V_{1}(x ; b)$ to be the expected discounted dividends until ruin when the time to the next dividend decision has the same distribution as $T_{1}+T_{2}$ (i.e. immediately after a dividend decision), where $x$ is the initial surplus and $b$ is the barrier level. Clearly, one has $V(x ; b)=V_{1}(x ; b)$. Similarly, for $x \geq 0$ we let $V_{2}(x ; b)$ be the dividend function when the time to the next dividend decision is instead distributed as $T_{1}$ (i.e. at an observation time that is not a dividend decision time). Because $V_{2}(x ; b)$ behaves differently on $0 \leq x \leq b$ and $x>b$, it will be useful define $V_{2}(x ; b)$ as a piecewise function, namely

$$
V_{2}(x ; b)= \begin{cases}V_{2, L}(x ; b), & 0 \leq x \leq b \\ V_{2, U}(x ; b), & x>b\end{cases}
$$


where the subscripts ' $L$ ' and ' $U$ ' represent 'lower' and 'upper' layer respectively.

The discounted densities $g_{\delta,+}(\cdot)$ and $g_{\delta,-}(\cdot)$ defined via (1.3) and (1.4) can now be applied to derive integral equations as follows. First, by conditioning on the pair $\left(T_{1}, \sum_{i=1}^{N\left(T_{1}\right)} Y_{i}-c T_{1}\right)$ we arrive at

$$
\begin{aligned}
V_{1}(x ; b)= & \int_{b-x}^{\infty} g_{\delta,-}(y) V_{2, U}(x+y ; b) d y+\int_{0}^{b-x} g_{\delta,-}(y) V_{2, L}(x+y ; b) d y \\
& +\int_{0}^{x} g_{\delta,+}(y) V_{2, L}(x-y ; b) d y, \quad 0 \leq x \leq b .
\end{aligned}
$$

Similarly, $V_{2, L}(x ; b)$ and $V_{2, U}(x ; b)$ can be respectively expressed as

$$
\begin{aligned}
V_{2, L}(x ; b)= & \int_{b-x}^{\infty} g_{\delta,-}(y)\left[y-(b-x)+V_{1}(b ; b)\right] d y+\int_{0}^{b-x} g_{\delta,-}(y) V_{1}(x+y ; b) d y \\
& +\int_{0}^{x} g_{\delta,+}(y) V_{1}(x-y ; b) d y, \quad 0 \leq x \leq b, \\
V_{2, U}(x ; b)= & \int_{0}^{\infty} g_{\delta,-}(y)\left[x+y-b+V_{1}(b ; b)\right] d y+\int_{0}^{x-b} g_{\delta,+}(y)\left[x-y-b+V_{1}(b ; b)\right] d y \\
& +\int_{x-b}^{x} g_{\delta,+}(y) V_{1}(x-y ; b) d y, \quad x>b .
\end{aligned}
$$

Under the present distributional assumptions, it is known from Albrecher et al. (2011b, Section 3.1) that

$$
g_{\delta,-}(y)=\frac{\gamma\left(\nu+\rho_{\gamma}\right)}{c \rho_{\gamma}\left(\rho_{\gamma}+R_{\gamma}\right)} \rho_{\gamma} e^{-\rho_{\gamma} y} \quad \text { and } \quad g_{\delta,+}(y)=\frac{\gamma\left(\nu-R_{\gamma}\right)}{c R_{\gamma}\left(\rho_{\gamma}+R_{\gamma}\right)} R_{\gamma} e^{-R_{\gamma} y}, \quad y>0
$$

are (defective) exponential densities, where $\rho_{\gamma}>0$ and $-R_{\gamma}<0$ are the roots of the quadratic equation (in $\xi$ )

$$
\xi^{2}+\left(\nu-\frac{\lambda+\gamma+\delta}{c}\right) \xi-\frac{(\gamma+\delta) \nu}{c}=0
$$

By substituting (2.4) into (2.1)-(2.3) and then applying the operator $\left(d / d x-\rho_{\gamma}\right)\left(d / d x+R_{\gamma}\right)$ on both sides of these equations, one can obtain a system of second-order differential equations in $x$ with constant coefficients, namely

$$
\begin{aligned}
V_{1}^{\prime \prime}+\left(R_{\gamma}-\rho_{\gamma}\right) V_{1}^{\prime}-\rho_{\gamma} R_{\gamma} V_{1} & =-\frac{\gamma}{c} V_{2, L}^{\prime}-\frac{\nu \gamma}{c} V_{2, L}, \quad 0 \leq x \leq b, \\
V_{2, L}^{\prime \prime}+\left(R_{\gamma}-\rho_{\gamma}\right) V_{2, L}^{\prime}-\rho_{\gamma} R_{\gamma} V_{2, L} & =-\frac{\gamma}{c} V_{1}^{\prime}-\frac{\nu \gamma}{c} V_{1}, \quad 0 \leq x \leq b, \\
V_{2, U}^{\prime \prime}+\left(R_{\gamma}-\rho_{\gamma}\right) V_{2, U}^{\prime}-\rho_{\gamma} R_{\gamma} V_{2, U} & =-\frac{\gamma}{c}\left[1+\nu\left(x-b+V_{1}(b ; b)\right)\right], \quad x>b .
\end{aligned}
$$

For convenience we have suppressed the dependence of $V_{1}, V_{2, L}$ and $V_{2, U}$ on $x$ and $b$, and it is understood that any derivatives are taken with respect to $x$ unless specified otherwise. To determine the solution, we first rewrite (2.5) and (2.6) in the form of the first-order homogeneous matrix differential equation

$$
\overrightarrow{\mathbf{V}}^{\prime}=\mathbf{A} \overrightarrow{\mathbf{V}}, \quad 0 \leq x \leq b,
$$

where $\overrightarrow{\mathbf{V}}=\left(V_{1}, V_{2, L}, V_{1}^{\prime}, V_{2, L}^{\prime}\right)^{\top}$ and

$$
\mathbf{A}=\left[\begin{array}{cccc}
0 & 0 & 1 & 0 \\
0 & 0 & 0 & 1 \\
\rho_{\gamma} R_{\gamma} & -\frac{\nu \gamma}{c} & \rho_{\gamma}-R_{\gamma} & -\frac{\gamma}{c} \\
-\frac{\nu \gamma}{c} & \rho_{\gamma} R_{\gamma} & -\frac{\gamma}{c} & \rho_{\gamma}-R_{\gamma}
\end{array}\right]
$$


Because the solution of (2.8) can be expressed in terms of a matrix exponential, this immediately gives

$$
\begin{aligned}
V_{1}(x ; b) & =C_{1} e^{\alpha_{1} x}+C_{2} e^{\alpha_{2} x}+C_{3} e^{\alpha_{3} x}+C_{4} e^{\alpha_{4} x}, & 0 \leq x \leq b, \\
V_{2, L}(x ; b) & =D_{1} e^{\alpha_{1} x}+D_{2} e^{\alpha_{2} x}+D_{3} e^{\alpha_{3} x}+D_{4} e^{\alpha_{4} x}, & 0 \leq x \leq b,
\end{aligned}
$$

where $\left\{C_{i}\right\}_{i=1}^{4}$ and $\left\{D_{i}\right\}_{i=1}^{4}$ are constants to be determined, and $\left\{\alpha_{i}\right\}_{i=1}^{4}$ are the eigenvalues of $\mathbf{A}$. For (2.7), the only unknown function in $x$ is $V_{2, U}(x ; b)$. Clearly, $\rho_{\gamma}$ and $-R_{\gamma}$ are roots of the characteristic equation pertaining to the homogeneous version of (2.7). Due to the non-homogeneous term which is linear in $x$, the general solution is therefore

$$
V_{2, U}(x ; b)=E_{1} x+E_{2}+E_{3} e^{-R_{\gamma} x}+E_{4} e^{\rho_{\gamma} x}, \quad x>b,
$$

for some constants $\left\{E_{i}\right\}_{i=1}^{4}$. Noting that all the dividend functions under consideration cannot exceed $x+c / \delta$ (i.e. the present value of insurer's payout cannot exceed the entire surplus plus the perpetuity of premium income), the linear boundedness implies $E_{4}=0$ by taking the limit $x \rightarrow \infty$ in (2.11).

With the solution forms (2.9)-(2.11) identified, it remains to determine the unknown constants. We proceed by plugging (2.9)-(2.11) as well as the discounted density (2.4) into the integral equations (2.1)(2.3). Omitting the straightforward details, equating the coefficients of $e^{\alpha_{i} x}$ in the substituted equation (2.1) yields

$$
C_{i}=D_{i} Z\left(\alpha_{i}\right), \quad i=1,2,3,4,
$$

where

$$
Z(s)=\frac{\gamma\left(\nu+\rho_{\gamma}\right)}{c\left(\rho_{\gamma}+R_{\gamma}\right)} \frac{1}{\rho_{\gamma}-s}+\frac{\gamma\left(\nu-R_{\gamma}\right)}{c\left(\rho_{\gamma}+R_{\gamma}\right)} \frac{1}{R_{\gamma}+s} .
$$

Similarly, equating the coefficients of $e^{\alpha_{i} x}$ in the substituted equation (2.2) gives

$$
D_{i}=C_{i} Z\left(\alpha_{i}\right), \quad i=1,2,3,4 .
$$

Combining (2.12) and (2.14), we arrive at

$$
Z\left(\alpha_{i}\right)= \pm 1, \quad i=1,2,3,4 .
$$

According to the discussion following equation (30) in Albrecher et al. (2011b), the roots of the equation (in $\xi) Z(\xi)=1$ are $\rho_{0}$ and $-R_{0}$. On the other hand, by application of the Vieta's rule concerning the roots $\rho_{\gamma}$ and $-R_{\gamma}$, it can be shown that $Z(\xi)=-1$ is equivalent to the quadratic equation (in $\xi$ )

$$
\xi^{2}+\left(\nu-\frac{\lambda+2 \gamma+\delta}{c}\right) \xi-\frac{(2 \gamma+\delta) \nu}{c}=0,
$$

for which the roots are $\rho_{2 \gamma}$ and $-R_{2 \gamma}$. Hence, we set

$$
\alpha_{1}=\rho_{0}, \quad \alpha_{2}=-R_{0}, \quad \alpha_{3}=\rho_{2 \gamma}, \quad \alpha_{4}=-R_{2 \gamma} .
$$

Incorporating (2.15) and (2.16) into (2.12) (or (2.14)) gives

$$
C_{1}=D_{1}, \quad C_{2}=D_{2}, \quad C_{3}=-D_{3}, \quad C_{4}=-D_{4} .
$$

We now return to the task of equating the coefficients of various exponential terms. For the substituted equation (2.1), matching the coefficients of $e^{-R_{\gamma} x}$ and $e^{\rho_{\gamma} x}$ respectively leads to

$$
\begin{aligned}
& \sum_{i=1}^{4} \frac{D_{i}}{R_{\gamma}+\alpha_{i}}=0 \\
& \sum_{i=1}^{4} \frac{D_{i} \rho_{\gamma} e^{\alpha_{i} b}}{\rho_{\gamma}-\alpha_{i}}=E_{2}+E_{1}\left(b+\frac{1}{\rho_{\gamma}}\right)+E_{3}\left(\frac{\rho_{\gamma} e^{-R_{\gamma} b}}{\rho_{\gamma}+R_{\gamma}}\right) .
\end{aligned}
$$


For the substituted equation (2.2), equating the coefficients of $e^{-R_{\gamma} x}$ and $e^{\rho_{\gamma} x}$ respectively results in

$$
\begin{aligned}
& \sum_{i=1}^{4} \frac{C_{i}}{R_{\gamma}+\alpha_{i}}=0, \\
& \sum_{i=1}^{4} \frac{C_{i} \alpha_{i} e^{\alpha_{i} b}}{\rho_{\gamma}-\alpha_{i}}=\frac{1}{\rho_{\gamma}} .
\end{aligned}
$$

Finally, for the substituted equation (2.3), equating the coefficients of $x$, the constant term and the coefficients of $e^{-R_{\gamma} x}$ produces respectively

$$
\begin{aligned}
& E_{1}=\frac{\gamma}{\gamma+\delta}, \\
& E_{2}=\frac{\gamma}{\gamma+\delta}\left(V_{1}(b ; b)-b\right)+\frac{\gamma\left(\nu+\rho_{\gamma}\right)}{c \rho_{\gamma}^{2}\left(\rho_{\gamma}+R_{\gamma}\right)}-\frac{\gamma\left(\nu-R_{\gamma}\right)}{c R_{\gamma}^{2}\left(\rho_{\gamma}+R_{\gamma}\right)}, \\
& E_{3}=\frac{\gamma\left(\nu-R_{\gamma}\right)}{c R_{\gamma}\left(\rho_{\gamma}+R_{\gamma}\right)}\left(\frac{e^{R_{\gamma} b}}{R_{\gamma}}-\sum_{i=1}^{4} \frac{C_{i} \alpha_{i} e^{\alpha_{i} b+R_{\gamma} b}}{R_{\gamma}+\alpha_{i}}\right) .
\end{aligned}
$$

Utilizing (2.9) at $x=b,(2.17)$ as well as (2.22)-(2.24), one can rewrite (2.18) and (2.19) as formulas involving only $\left\{C_{i}\right\}_{i=1}^{4}$ as unknown constants. These together with (2.20) and (2.21) form a system of four linear equations that can be used to calculate $\left\{C_{i}\right\}_{i=1}^{4}$ (and hence $\left\{D_{i}\right\}_{i=1}^{4}, E_{2}$ and $E_{3}$ ). Therefore, the values of $V_{1}$ and $V_{2}$ can be computed using (2.9)-(2.11), where $\left\{\alpha_{i}\right\}_{i=1}^{4}$ are given by (2.16).

\subsection{Model with $j \in \mathbb{N}$}

Now we aim at extending the results in the previous subsection to the case of integer $j \in \mathbb{N}$. With $x$ being the initial surplus and $b$ the barrier level, for $i=1,2, \ldots, j$, we define $V_{i}(x ; b)$ to be the expected discounted dividends until ruin when the time to the next dividend decision is distributed as $\sum_{k=1}^{j-i+1} T_{k}$. While $V(x ; b)=V_{1}(x ; b)$ is only defined when $0 \leq x \leq b$, the function $V_{i}(x ; b)$ is defined in a piecewise manner for $i=2,3, \ldots, j$, namely

$$
V_{i}(x ; b)= \begin{cases}V_{i, L}(x ; b), & 0 \leq x \leq b . \\ V_{i, U}(x ; b), & x>b .\end{cases}
$$

Concerning $V_{1}(x ; b)$, it is easy to see that the integral equation $(2.1)$ still holds true. For $i=2,3 \ldots, j-$ 1 , the dividend functions $V_{i, L}(x ; b)$ and $V_{i, U}(x ; b)$ respectively satisfy

$$
\begin{aligned}
V_{i, L}(x ; b)= & \int_{b-x}^{\infty} g_{\delta,-}(y) V_{i+1, U}(x+y ; b) d y+\int_{0}^{b-x} g_{\delta,-}(y) V_{i+1, L}(x+y ; b) d y \\
& +\int_{0}^{x} g_{\delta,+}(y) V_{i+1, L}(x-y ; b) d y, \quad 0 \leq x \leq b, \\
V_{i, U}(x ; b)= & \int_{x-b}^{x} g_{\delta,+}(y) V_{i+1, L}(x-y ; b) d y+\int_{0}^{x-b} g_{\delta,+}(y) V_{i+1, U}(x-y ; b) d y \\
& +\int_{0}^{\infty} g_{\delta,-}(y) V_{i+1, U}(x+y ; b) d y, \quad x>b .
\end{aligned}
$$

Then, for $V_{j}(x ; b)$, the same technique gives rise to

$$
V_{j, L}(x ; b)=\int_{b-x}^{\infty} g_{\delta,-}(y)\left[y-(b-x)+V_{1}(b ; b)\right] d y+\int_{0}^{b-x} g_{\delta,-}(y) V_{1}(x+y ; b) d y
$$




$$
\begin{aligned}
& +\int_{0}^{x} g_{\delta,+}(y) V_{1}(x-y ; b) d y, \quad 0 \leq x \leq b, \\
V_{j, U}(x ; b)= & \int_{0}^{\infty} g_{\delta,-}(y)\left[x+y-b+V_{1}(b ; b)\right] d y+\int_{0}^{x-b} g_{\delta,+}(y)\left[x-y-b+V_{1}(b ; b)\right] d y \\
& +\int_{x-b}^{x} g_{\delta,+}(y) V_{1}(x-y ; b) d y, \quad x>b .
\end{aligned}
$$

Remark 2.1 By putting $x=b^{-}$into (2.25) and (2.27) as well as $x=b^{+}$into (2.26) and (2.28), it is clear that for $i=2,3, \ldots, j$ one has $V_{i, L}\left(b^{-} ; b\right)=V_{i, U}\left(b^{+} ; b\right)$, i.e. $V_{i}(x ; b)$ is continuous at $x=b$.

Next, with (2.4) in mind, application of the operator $\left(d / d x-\rho_{\gamma}\right)\left(d / d x+R_{\gamma}\right)$ to $(2.1)$ and (2.25)-(2.28) leads to a system of second-order differential equations in $x$ with constant coefficients given by

$$
\begin{aligned}
V_{1}^{\prime \prime}+\left(R_{\gamma}-\rho_{\gamma}\right) V_{1}^{\prime}-\rho_{\gamma} R_{\gamma} V_{1} & =-\frac{\gamma}{c} V_{2, L}^{\prime}-\frac{\nu \gamma}{c} V_{2, L}, \quad 0 \leq x \leq b, \\
V_{i, L}^{\prime \prime}+\left(R_{\gamma}-\rho_{\gamma}\right) V_{i, L}^{\prime}-\rho_{\gamma} R_{\gamma} V_{i, L} & =-\frac{\gamma}{c} V_{i+1, L}^{\prime}-\frac{\nu \gamma}{c} V_{i+1, L}, \quad i=2,3, \ldots, j-1 ; \quad 0 \leq x \leq b, \\
V_{i, U}^{\prime \prime}+\left(R_{\gamma}-\rho_{\gamma}\right) V_{i, U}^{\prime}-\rho_{\gamma} R_{\gamma} V_{i, U} & =-\frac{\gamma}{c} V_{i+1, U}^{\prime}-\frac{\nu \gamma}{c} V_{i+1, U}, \quad i=2,3, \ldots, j-1 ; \quad x>b, \\
V_{j, L}^{\prime \prime}+\left(R_{\gamma}-\rho_{\gamma}\right) V_{j, L}^{\prime}-\rho_{\gamma} R_{\gamma} V_{j, L} & =-\frac{\gamma}{c} V_{1}^{\prime}-\frac{\nu \gamma}{c} V_{1}, \quad 0 \leq x \leq b, \\
V_{j, U}^{\prime \prime}+\left(R_{\gamma}-\rho_{\gamma}\right) V_{j, U}^{\prime}-\rho_{\gamma} R_{\gamma} V_{j, U} & =-\frac{\gamma}{c}\left[1+\nu\left(x-b+V_{1}(b ; b)\right)\right], \quad x>b .
\end{aligned}
$$

The equations (2.29), (2.30) and (2.32) can be collectively rewritten as the first-order homogeneous matrix differential equation (2.8) with the extended definitions $\overrightarrow{\mathbf{V}}=\left(V_{1}, V_{2, L}, \ldots, V_{j, L}, V_{1}^{\prime}, V_{2, L}^{\prime}, \ldots, V_{j, L}^{\prime}\right)^{\top}$ and

$$
\mathbf{A}=\left[\begin{array}{cc}
\mathbf{0} & \mathbf{I} \\
\mathbf{M}_{1} & \mathbf{M}_{2}
\end{array}\right]
$$

where $\mathbf{0}$ and $\mathbf{I}$ are the $j \times j$ zero and identity matrices respectively, and $\mathbf{M}_{1}$ and $\mathbf{M}_{2}$ are the $j$-dimensional square matrices

$$
\mathbf{M}_{1}=\left[\begin{array}{cccc}
\rho_{\gamma} R_{\gamma} & -\frac{\nu \gamma}{c} & 0 & \cdots \\
0 & \rho_{\gamma} R_{\gamma} & -\frac{\nu \gamma}{c} & 0 \\
\vdots & \ddots & \ddots & \ddots \\
-\frac{\nu \gamma}{c} & 0 & 0 & \rho_{\gamma} R_{\gamma}
\end{array}\right] \quad \text { and } \quad \mathbf{M}_{2}=\left[\begin{array}{cccc}
\rho_{\gamma}-R_{\gamma} & -\frac{\gamma}{c} & 0 & \ldots \\
0 & \rho_{\gamma}-R_{\gamma} & -\frac{\gamma}{c} & 0 \\
\vdots & \ddots & \ddots & \ddots \\
-\frac{\gamma}{c} & 0 & 0 & \rho_{\gamma}-R_{\gamma}
\end{array}\right] \text {. }
$$

Using the same arguments leading to (2.9) and (2.10), one has that

$$
\begin{aligned}
& V_{1}(x ; b)=\sum_{k=1}^{2 j} C_{k, 1} e^{\alpha_{k} x}, \quad 0 \leq x \leq b, \\
& V_{i, L}(x ; b)=\sum_{k=1}^{2 j} C_{k, i} e^{\alpha_{k} x}, \quad i=2,3, \ldots, j ; \quad 0 \leq x \leq b,
\end{aligned}
$$

where $\left\{C_{k, i}\right\}_{k=1}^{2 j}$ for $i=1,2 \ldots, j$ are some constants to be determined and $\left\{\alpha_{k}\right\}_{k=1}^{2 j}$ are the eigenvalues of $\mathbf{A}$. As for (2.33), similar to (2.11) along with linear boundedness, the general solution is

$$
V_{j, U}(x ; b)=E_{1, j} x+E_{2, j}+E_{3, j} e^{-R_{\gamma} x}, \quad x>b,
$$


for some constants $\left\{E_{k, j}\right\}_{k=1}^{3}$. Concerning $V_{i, U}(x ; b)$ for $i=2,3, \ldots, j-1$, inductively it can be deduced from (2.31) that the non-homogeneous terms involve $x, e^{-R_{\gamma} x}, x e^{-R_{\gamma} x}, \ldots, x^{j-i-1} e^{-R_{\gamma} x}$ where these terms come from $V_{i+1, U}$ and $V_{i+1, U}^{\prime}$ (note also that $\rho_{\gamma}$ and $-R_{\gamma}$ are the roots of the characteristic equation of the homogeneous version of (2.31)). Along with the linear boundedness of $V_{i, U}$, the general solution is thus

$$
V_{i, U}(x ; b)=E_{1, i} x+E_{2, i}+\sum_{l=0}^{j-i} E_{l+3, i} x^{l} e^{-R_{\gamma} x}, \quad i=2,3, \ldots, j-1 ; \quad x>b,
$$

for some constants $\left\{E_{k, i}\right\}_{k=1}^{j-i+3}$.

The remaining task is to determine the unknown constants involved in (2.34)-(2.37). This is done by back substituting these solutions and (2.4) into (2.1) and (2.25)-(2.28) and then performing straightforward but tedious integrations. Equating the coefficients of $e^{\alpha_{k} x}$ in the substituted equations (2.1) and (2.25) yields

$$
C_{k, i}=C_{k, i+1} Z\left(\alpha_{k}\right), \quad i=1,2, \ldots, j-1 ; \quad k=1,2 \ldots, 2 j,
$$

where $Z(\cdot)$ is defined in (2.13). Next, equating the coefficients of $e^{\alpha_{k} x}$ in the substituted equation (2.27) leads to

$$
C_{k, j}=C_{k, 1} Z\left(\alpha_{k}\right), \quad k=1,2, \ldots, 2 j .
$$

Using (2.38), (2.39) and de Moivre's formula, it can be observed that

$$
Z\left(\alpha_{k}\right)=e^{\mathrm{i} \frac{2 l \pi}{j}}, \quad l=0,1, \ldots, j-1 ; \quad k=1,2, \ldots, 2 j,
$$

where $\mathrm{i}$ is the unit imaginary number. For each $l=0,1, \ldots, j-1,(2.40)$ is a quadratic equation (in $\alpha_{k}$ ), and hence one concludes that $\left\{\alpha_{k}\right\}_{k=1}^{2 j}$ are the $2 j$ roots of (2.40). For the substituted equations (2.1), (2.25) and (2.27), by equating the coefficients of $e^{-R_{\gamma} x}$ and $e^{\rho_{\gamma} x}$ we arrive at

$$
\begin{aligned}
\sum_{k=1}^{2 j} \frac{C_{k, i}}{R_{\gamma}+\alpha_{k}} & =0, \quad i=1,2, \ldots, j, \\
\sum_{k=1}^{2 j} \frac{C_{k, 1} \alpha_{k} e^{\alpha_{k} b}}{\rho_{\gamma}-\alpha_{k}} & =\frac{1}{\rho_{\gamma}}, \\
\sum_{k=1}^{2 j} \frac{C_{k, i} \rho_{\gamma} e^{\alpha_{k} b}}{\rho_{\gamma}-\alpha_{k}} & =E_{2, i}+\left(b+\frac{1}{\rho_{\gamma}}\right) E_{1, i}+\rho_{\gamma} e^{-R_{\gamma} b} \sum_{l=0}^{j-i} \sum_{m=0}^{l} \frac{E_{l+3, i} l ! b^{m}}{m !\left(\rho_{\gamma}+R_{\gamma}\right)^{l+1-m}}, \quad i=2,3, \ldots, j .
\end{aligned}
$$

For the substituted equation (2.28), the terms involving $x$, the constants and the coefficients of $e^{-R_{\gamma} x}$ respectively imply

$$
\begin{aligned}
& E_{1, j}=\frac{\gamma}{\gamma+\delta}, \\
& E_{2, j}=\frac{\gamma}{\gamma+\delta}\left(V_{1}(b ; b)-b\right)+\frac{\gamma\left(\nu+\rho_{\gamma}\right)}{c \rho_{\gamma}^{2}\left(\rho_{\gamma}+R_{\gamma}\right)}-\frac{\gamma\left(\nu-R_{\gamma}\right)}{c R_{\gamma}^{2}\left(\rho_{\gamma}+R_{\gamma}\right)}, \\
& E_{3, j}=\frac{\gamma\left(\nu-R_{\gamma}\right)}{c R_{\gamma}\left(\rho_{\gamma}+R_{\gamma}\right)}\left(\frac{e^{R_{\gamma} b}}{R_{\gamma}}-\sum_{k=1}^{2 j} \frac{C_{k, 1} \alpha_{k} e^{\alpha_{k} b+R_{\gamma} b}}{R_{\gamma}+\alpha_{k}}\right) .
\end{aligned}
$$

For the substituted equation (2.26), equating the coefficients of $x$, the constant term and the coefficients of $x^{l} e^{-R_{\gamma} x}$ (with $l=0,1 \ldots, j-i$ ) gives rise to, for $i=2,3, \ldots, j-1$, 


$$
\begin{aligned}
E_{1, i}= & \left(\frac{\gamma}{\gamma+\delta}\right) E_{1, i+1} \\
E_{2, i}= & \left(\frac{\gamma}{\gamma+\delta}\right) E_{2, i+1}+\left(\frac{\gamma\left(\nu+\rho_{\gamma}\right)}{c \rho_{\gamma}^{2}\left(\rho_{\gamma}+R_{\gamma}\right)}-\frac{\gamma\left(\nu-R_{\gamma}\right)}{c R_{\gamma}^{2}\left(\rho_{\gamma}+R_{\gamma}\right)}\right) E_{1, i+1}, \\
E_{3, i}= & \frac{\gamma\left(\nu-R_{\gamma}\right)}{c R_{\gamma}\left(\rho_{\gamma}+R_{\gamma}\right)}\left(\sum_{k=1}^{2 j} \frac{C_{k, i+1} R_{\gamma} e^{\alpha_{k} b+R_{\gamma} b}}{R_{\gamma}+\alpha_{k}}-E_{1, i+1} e^{R_{\gamma} b}\left(b-\frac{1}{R_{\gamma}}\right)\right. \\
& \left.-E_{2, i+1} e^{R_{\gamma} b}-R_{\gamma} \sum_{l=0}^{j-i-1} \frac{E_{l+3, i+1} b^{l+1}}{l+1}\right)+\frac{\gamma\left(\nu+\rho_{\gamma}\right)}{c\left(\rho_{\gamma}+R_{\gamma}\right)} \sum_{l=0}^{j-i-1} \frac{E_{l+3, i+1} l !}{\left(\rho_{\gamma}+R_{\gamma}\right)^{l+1}}, \\
E_{l+3, i}= & \left(\frac{\gamma\left(\nu-R_{\gamma}\right)}{c l\left(\rho_{\gamma}+R_{\gamma}\right)}\right) E_{l+2, i+1}+\frac{\gamma\left(\nu+\rho_{\gamma}\right)}{c\left(\rho_{\gamma}+R_{\gamma}\right)} \sum_{m=l}^{j-i-1} \frac{E_{m+3, i+1} m !}{l !\left(\rho_{\gamma}+R_{\gamma}\right)^{m-l+1}}, \quad l=1,2, \ldots, j-i .
\end{aligned}
$$

Note that the recursive relationships (2.47) and (2.48) for $E_{1, i}$ and $E_{2, i}$ with starting points (2.44) and (2.45) can be solved as, for $i=2,3, \ldots, j$,

$$
\begin{aligned}
& E_{1, i}=\left(\frac{\gamma}{\gamma+\delta}\right)^{j-i+1}, \\
& E_{2, i}=\left(\frac{\gamma}{\gamma+\delta}\right)^{j-i+1}\left(V_{1}(b ; b)-b\right)+(j-i+1)\left(\frac{\gamma}{\gamma+\delta}\right)^{j-i}\left(\frac{\gamma\left(\nu+\rho_{\gamma}\right)}{c \rho_{\gamma}^{2}\left(\rho_{\gamma}+R_{\gamma}\right)}-\frac{\gamma\left(\nu-R_{\gamma}\right)}{c R_{\gamma}^{2}\left(\rho_{\gamma}+R_{\gamma}\right)}\right) .
\end{aligned}
$$

Moreover, using (2.38) and (2.39), one can express $C_{k, i}$ in terms of $C_{k, 1}$ as

$$
C_{k, i}=Z\left(\alpha_{k}\right)^{1-i} C_{k, 1}, \quad i=1,2, \ldots, j ; \quad k=1,2 \ldots, 2 j .
$$

To summarize, plugging (2.53) into (2.41) results in $j$ equations in terms of the unknowns $\left\{C_{k, 1}\right\}_{k=1}^{2 j}$. In addition, for each $i=2,3, \ldots, j$, with $E_{1, i}$ explicitly known in (2.51), it can be seen from (2.46), (2.49), (2.50) and (2.52) along with (2.34) at $x=b$ that the constants $\left\{E_{k, i}\right\}_{k=2}^{j-i+3}$ depend on $\left\{C_{k, 1}\right\}_{k=1}^{2 j}$ in a linear manner. Thus, we have another set of $j$ equations from (2.42) and (2.43), leading to a total of $2 j$ equations in the $2 j$ unknowns $\left\{C_{k, 1}\right\}_{k=1}^{2 j}$. With $\left\{C_{k, 1}\right\}_{k=1}^{2 j}$ solved and $\left\{\alpha_{k}\right\}_{k=1}^{2 j}$ obtained as the $2 j$ roots of (2.40), for $i=2,3, \ldots, j$ the values of $\left\{C_{k, i}\right\}_{k=1}^{2 j}$ and $\left\{E_{k, i}\right\}_{k=1}^{j-i+3}$ can be calculated accordingly. Then the dividend functions (2.34)-(2.37) are fully characterized.

\section{Claims with rational Laplace transform and Erlang inter-observation times}

In this section, we shall generalize the results in Section 2.2 concerning $j \in \mathbb{N}$ under fairly general distributional assumptions. Although the resulting formulas are very lengthy, with the Erlangization technique in mind they are included for the sake of completeness. In what follows, the inter-observation times are assumed to follow $\operatorname{Erlang}(n)$ distribution with density (1.2) while the claims have rational Laplace transform, i.e.

$$
\tilde{f}_{Y}(s)=\frac{Q_{2, r-1}(s)}{Q_{1, r}(s)}
$$

Here $Q_{1, r}(\cdot)$ is a polynomial of degree $r$ with leading coefficient 1 , and $Q_{2, r-1}(\cdot)$ is a polynomial of degree at most $r-1$ having no common zeros with $Q_{1, r}(\cdot)$. It is known from Albrecher et al. (2013, Section 4.1) that the discounted densities $g_{\delta,+}(\cdot)$ and $g_{\delta,-}(\cdot)$ in (1.4) are given by 


$$
g_{\delta,-}(y)=\sum_{j=1}^{n} B_{j}^{*} \frac{y^{j-1} e^{-\rho_{\gamma} y}}{(j-1) !} \quad \text { and } \quad g_{\delta,+}(y)=\sum_{i=1}^{r} \sum_{j=1}^{n} B_{i j} \frac{y^{j-1} e^{-R_{\gamma, i} y}}{(j-1) !}, \quad y>0 .
$$

In the above expressions, $\rho_{\gamma}>0$ is the unique positive root of the equation (in $\xi$ )

$$
c \xi-(\lambda+\gamma+\delta)+\lambda \widetilde{f}_{Y}(\xi)=0
$$

and $\left\{-R_{\gamma, i}\right\}_{i=1}^{r}$ are the remaining $r$ roots which have negative real parts. The constants $B_{j}^{*}$ 's and $B_{i j}$ 's are given by

$$
B_{j}^{*}=\left.(-1)^{n-j}\left(\frac{\gamma}{c}\right)^{n} \frac{1}{(n-j) !} \frac{d^{n-j}}{d s^{n-j}} \frac{\left[Q_{1, r}(s)\right]^{n}}{\prod_{l=1}^{r}\left(s+R_{\gamma, l}\right)^{n}}\right|_{s=\rho_{\gamma}}, \quad j=1,2, \ldots, n,
$$

and

$$
B_{i j}=\left.\left(\frac{\gamma}{c}\right)^{n} \frac{1}{(n-j) !} \frac{d^{n-j}}{d s^{n-j}} \frac{\left[Q_{1, r}(s)\right]^{n}}{\left(\rho_{\gamma}-s\right)^{n} \prod_{l=1, l \neq i}^{r}\left(s+R_{\gamma, l}\right)^{n}}\right|_{s=-R_{\gamma, i}}, \quad i=1,2, \ldots, r ; \quad j=1,2, \ldots, n .
$$

We begin our analysis by noting that the integral equations (2.1) and (2.25)-(2.28) remain valid in this general case with (3.2) in place. By applying the operator $\left(d / d x-\rho_{\gamma}\right)^{n} \prod_{l=1}^{r}\left(d / d x+R_{\gamma, l}\right)^{n}$ to $(2.1),(2.25)$ and (2.27), we obtain a system of $j$ homogeneous differential equations satisfied by $\left(V_{1}, V_{2, L}, \ldots, V_{j, L}\right)$, each of which is of order $n(r+1)$ and has constant coefficients. Therefore, the solution forms of $V_{1}$ and $\left\{V_{i, L}\right\}_{i=2}^{j}$ are given by

$$
\begin{aligned}
& V_{1}(x ; b)=\sum_{k=1}^{j n(r+1)} C_{k, 1} e^{\alpha_{k} x}, \quad 0 \leq x \leq b, \\
& V_{i, L}(x ; b)=\sum_{k=1}^{j n(r+1)} C_{k, i} e^{\alpha_{k} x}, \quad i=2,3, \ldots, j ; \quad 0 \leq x \leq b,
\end{aligned}
$$

for some constants $\left\{C_{k, i}\right\}_{k=1}^{j n(r+1)}(i=1,2, \ldots, j)$ and $\left\{\alpha_{k}\right\}_{k=1}^{j n(r+1)}$. Application of the same operator $\left(d / d x-\rho_{\gamma}\right)^{n} \prod_{l=1}^{r}\left(d / d x+R_{\gamma, l}\right)^{n}$ to $(2.28)$ gives rise to an $n(r+1)$-th order differential equation in $V_{j, U}$ with constant coefficients, where the non-homogeneous term is linear in $x$. The associated characteristic equation has roots $\rho_{\gamma}$ and $\left\{-R_{\gamma, l}\right\}_{l=1}^{r}$, each with multiplicity $n$. Thus, $V_{j, U}$ can be expressed as

$$
V_{j, U}(x ; b)=E_{1, j} x+E_{2, j}+\sum_{l=1}^{r} \sum_{k=0}^{n-1} E_{k+3, j, l} x^{k} e^{-R_{\gamma, l} x}, \quad x>b,
$$

for some constants $E_{1, j}, E_{2, j}$ and $\left\{E_{k+3, j, l}\right\}_{k=0}^{n-1}(l=1,2, \ldots, r)$, where the terms in relation to $e^{\rho_{\gamma} x}$ vanish by linear boundedness. Similarly, we again apply $\left(d / d x-\rho_{\gamma}\right)^{n} \prod_{l=1}^{r}\left(d / d x+R_{\gamma, l}\right)^{n}$ to (2.26) for each fixed $i=2,3, \ldots, j-1$ and observe that $V_{i, U}$ satisfies the same differential equation as $V_{j, U}$ except that the non-homogeneous part now involves terms in $x$ and $x^{k} e^{-R_{\gamma, l} x}$ for $k=0,1, \ldots,(j-i) n-1$ and $l=1,2, \ldots, r$. Therefore, we arrive at

$$
V_{i, U}(x ; b)=E_{1, i} x+E_{2, i}+\sum_{l=1}^{r} \sum_{k=0}^{(j-i+1) n-1} E_{k+3, i, l} x^{k} e^{-R_{\gamma, l} x}, \quad i=2,3, \ldots, j-1 ; \quad x>b,
$$

for some constants $E_{1, i}, E_{2, i}$ and $\left\{E_{k+3, i, l}\right\}_{k=0}^{(j-i+1) n-1}(l=1,2 \ldots, r)$. 
To determine the above unknown constants, we first substitute (3.2) and (3.6)-(3.9) into (2.1), (2.25) and (2.27). Skipping the tedious details, by equating the coefficients of $e^{\alpha_{k} x}$ in these substituted equations, we arrive at the same equations as (2.38) and (2.39) with the domain of $k$ extended to $k=1,2, \ldots, j n(r+$ 1) and the definition of $Z(\cdot)$ generalized to

$$
Z(s)=\sum_{m=1}^{n} \frac{B_{m}^{*}}{\left(\rho_{\gamma}-s\right)^{m}}+\sum_{z=1}^{r} \sum_{m=1}^{n} \frac{B_{z m}}{\left(R_{\gamma, z}+s\right)^{m}} .
$$

Hence, one asserts that (2.40) holds true with $k=1,2, \ldots, j n(r+1)$. Because for each $l=0,1, \ldots, j-1$, (2.40) is now a polynomial (in $\alpha_{k}$ ) of degree $n(r+1)$, a total of $j n(r+1)$ roots, namely $\left\{\alpha_{k}\right\}_{k=1}^{j n(r+1)}$, arise. In addition, each $C_{k, i}$ can be expressed in terms of $C_{k, 1}$ as in (2.53), also with extended domain $k=1,2, \ldots, j n(r+1)$. Next, equating the coefficients of $x^{p-1} e^{-R_{\gamma, z} x}$ in the substituted (2.1), (2.25) and (2.27) leads to

$$
\sum_{k=1}^{j n(r+1)} \sum_{m=p}^{n} \frac{C_{k, i} B_{z m}}{\left(R_{\gamma, z}+\alpha_{k}\right)^{m-p+1}}=0, \quad i=1,2, \ldots, j ; \quad z=1,2, \ldots, r ; \quad p=1,2, \ldots, n .
$$

We also equate the coefficients of $x^{p} e^{\rho_{\gamma} x}$ in (2.1) and (2.25) to yield

$$
\begin{aligned}
\sum_{l=p}^{n-1} \sum_{m=l+1}^{n} \sum_{k=1}^{j n(r+1)} \frac{B_{m}^{*} C_{k, i+1} b^{l-p} e^{\alpha_{k} b}}{(l-p) !\left(\rho_{\gamma}-\alpha_{k}\right)^{m-l}}= & \sum_{m=p+1}^{n} \sum_{s=0}^{m-p} \frac{B_{m}^{*} E_{1, i+1}(m-p) b^{s}}{s ! \rho_{\gamma}^{m-p-s+1}}+\sum_{m=p+1}^{n} \sum_{s=0}^{m-p-1} \frac{B_{m}^{*} E_{2, i+1} b^{s}}{s ! \rho_{\gamma}^{m-p-s}} \\
& +\sum_{m=p+1}^{n} \sum_{k=0}^{(j-i) n-1} \sum_{l=1}^{r} \sum_{s=0}^{m-p+k-1} \frac{B_{m}^{*} E_{k+3, i+1, l} b^{s} e^{-R_{\gamma, l} b}(m-p-1+k) !}{s !(m-p-1) !\left(\rho_{\gamma}+R_{\gamma, l}\right)^{m-p+k-s}} \\
& i=1,2, \ldots, j-1 ; \quad p=0,1, \ldots, n-1 .
\end{aligned}
$$

For (2.27), matching the coefficients of $x^{p} e^{\rho_{\gamma} x}$ produces

$$
\begin{aligned}
\sum_{l=p}^{n-1} \sum_{m=l+1}^{n} \sum_{k=1}^{j n(r+1)} \frac{B_{m}^{*} C_{k, 1} b^{l} e^{\alpha_{k} b}}{(l-p) !\left(\rho_{\gamma}-\alpha_{k}\right)^{m-l}}+\sum_{s=p-1}^{n-1} \frac{\rho_{\gamma}^{s} b^{s+1}}{(s-(p-1)) !} \sum_{m=s+1}^{n} \frac{B_{m}^{*} p}{\rho_{\gamma}^{m}} \\
=\sum_{s=p}^{n-1} \frac{\rho_{\gamma}^{s} b^{s}}{(s-p) !} \sum_{m=s+1}^{n} \frac{B_{m}^{*}}{\rho_{\gamma}^{m}}\left(V_{1}(b ; b)-b+\frac{m}{\rho_{\gamma}}\right)+\sum_{m=p}^{n} \frac{B_{m}^{*} m b^{m}}{(m-p) ! \rho_{\gamma}}, \quad p=0,1, \ldots, n-1 .
\end{aligned}
$$

Now, we plug (3.2), (3.6) and (3.8) into (2.28). Equating the terms involving $x$, constants, $e^{-R_{\gamma, z} x}$ and $x^{p} e^{-R_{\gamma, z} x}$ gives

$$
\begin{aligned}
E_{1, j}= & \left(\frac{\gamma}{\gamma+\delta}\right)^{n}, \\
E_{2, j}= & \left(\frac{\gamma}{\gamma+\delta}\right)^{n}\left(V_{1}(b ; b)-b\right)+\sum_{m=1}^{n} \frac{B_{m}^{*} m}{\rho_{\gamma}^{m+1}}-\sum_{z=1}^{r} \sum_{m=1}^{n} \frac{B_{z m} m}{R_{\gamma, z}^{m+1}}, \\
E_{3, j, z}= & \sum_{s=0}^{n} \frac{R_{\gamma, z}^{s}(-b)^{s}}{s !} \sum_{m=s}^{n} \frac{B_{z m} m}{R_{\gamma, z}^{m+1}} e^{R_{\gamma, z} b}+\sum_{s=0}^{n-1} \frac{(-b)^{s}}{s !} \sum_{m=s+1}^{n} B_{z m} \sum_{k=1}^{j n(r+1)} \frac{C_{k, 1} e^{\alpha_{k} b}}{\left(R_{\gamma, z}+\alpha_{k}\right)^{m-s}} e^{R_{\gamma, z} b} \\
& -\sum_{s=0}^{n-1} \frac{R_{\gamma, z}^{s}(-b)^{s}}{s !} \sum_{m=s+1}^{n} \frac{B_{z m}}{R_{\gamma, z}^{m}}\left(V_{1}(b ; b)-b\right) e^{R_{\gamma, z} b}, \quad z=1,2, \ldots, r,
\end{aligned}
$$




$$
\begin{aligned}
E_{p+3, j, z}= & \sum_{s=p}^{n} \frac{R_{\gamma, z}^{s}(-b)^{s-p}}{(s-p) !} \sum_{m=s}^{n} \frac{B_{z m} m}{R_{\gamma, z}^{m+1}} \frac{e^{R_{\gamma, z} b}}{p !}+\sum_{s=p}^{n-1} \frac{(-b)^{s-p}}{(s-p) !} \sum_{m=s+1}^{n} B_{z m} \sum_{k=1}^{j n(r+1)} \frac{C_{k, 1} e^{\alpha_{k} b}}{\left(R_{\gamma, z}+\alpha_{k}\right)^{m-s}} \frac{e^{R_{\gamma, z} b}}{p !} \\
& -\sum_{s=p}^{n-1} \frac{R_{\gamma, z}^{s}(-b)^{s-p}}{(s-p) !} \sum_{m=s+1}^{n} \frac{B_{z m}}{R_{\gamma, z}^{m}}\left(V_{1}(b ; b)-b\right) \frac{e^{R_{\gamma, z} b}}{p !} \\
& -\sum_{s=p-1}^{n-1} \frac{R_{\gamma, z}^{s}(-b)^{s-p+1}}{(s-(p-1)) !} \sum_{m=s+1}^{n} \frac{B_{z m} p}{R_{\gamma, z}^{m}} \frac{e^{R_{\gamma, z} b}}{p !}, \quad z=1,2, \ldots, r ; \quad p=1,2, \ldots, n-1 .
\end{aligned}
$$

The next step is to substitute (3.2), (3.7) and (3.9) into (2.26). The terms involving $x$, constants, $e^{-R_{\gamma, z} x}$ and $x^{w} e^{-R_{\gamma, z} x}$ imply, for $i=2,3, \ldots, j-1$,

$$
\begin{aligned}
& E_{1, i}=\left(\frac{\gamma}{\gamma+\delta}\right)^{n} E_{1, i+1} \\
& E_{2, i}=\left(\frac{\gamma}{\gamma+\delta}\right)^{n} E_{2, i+1}+\left(\sum_{m=1}^{n} \frac{B_{m}^{*} m}{\rho_{\gamma}^{m+1}}-\sum_{z=1}^{r} \sum_{m=1}^{n} \frac{B_{z m} m}{R_{\gamma, z}^{m+1}}\right) E_{1, i+1}, \\
& E_{3, i, z}=\sum_{m=1}^{n} \frac{B_{m}^{*}}{(m-1) !} \sum_{k=0}^{(j-i) n-1} \frac{E_{k+3, i+1, z}(m+k-1) !}{\left(\rho_{\gamma}+R_{\gamma, z}\right)^{m+k}}+\sum_{s=0}^{n} \frac{R_{\gamma, z}^{s}(-b)^{s}}{s !} \sum_{m=s}^{n} \frac{B_{z m} m}{R_{\gamma, z}^{m+1}} E_{1, i+1} e^{R_{\gamma, z} b} \\
& -\sum_{s=0}^{n-1} \frac{R_{\gamma, z}^{s}(-b)^{s}}{s !} \sum_{m=s+1}^{n} \frac{B_{z m}}{R_{\gamma, z}^{m}} E_{2, i+1} e^{R_{\gamma, z} b}+\sum_{m=1}^{n} \frac{B_{z m}}{(m-1) !} \sum_{k=0}^{(j-i) n-1} \frac{E_{k+3, i+1, z}(-1)^{k}(-b)^{m+k}}{m+k} \\
& +\sum_{m=1}^{n} \sum_{l=1, l \neq z}^{r} \frac{B_{l m}}{(m-1) !} \sum_{k=0}^{(j-i) n-1} \frac{E_{k+3, i+1, z}(-1)^{k}(m+k-1) !}{\left(R_{\gamma, l}-R_{\gamma, z}\right)^{m+k}} \\
& +\sum_{l=1, l \neq z}^{r} e^{-R_{\gamma, l} b} \sum_{m=1}^{n} \frac{B_{z m}}{(m-1) !} \sum_{k=0}^{(j-i) n-1} E_{k+3, i+1, l}(-1)^{k+1}(m+k-1) ! \sum_{s=0}^{m+k-1} \frac{(-b)^{s}}{s !\left(R_{\gamma, z}-R_{\gamma, l}\right)^{m+k-s}} e^{R_{\gamma, z} b} \\
& +\sum_{s=0}^{n-1} \frac{(-b)^{s}}{s !} \sum_{m=s+1}^{n} B_{z m} \sum_{k=1}^{j n(r+1)} \frac{C_{k, i+1} e^{\alpha_{k} b}}{\left(R_{\gamma, z}+\alpha_{k}\right)^{m-s}} e^{R_{\gamma, z} b}, \quad z=1,2, \ldots, r, \\
& E_{w+3, i, z}=\sum_{m=1}^{n} \frac{B_{m}^{*}}{(m-1) !} \sum_{k=w}^{(j-i) n-1} \frac{E_{k+3, i+1, z}(m+k-w-1) !}{\left(\rho_{\gamma}+R_{\gamma, z}\right)^{m+k-w}}+\sum_{s=w}^{n} \frac{R_{\gamma, z}^{s}(-b)^{s-w}}{(s-w) !} \sum_{m=s}^{n} \frac{B_{z m m} m}{R_{\gamma, z}^{m+1}} \frac{E_{1, i+1} e^{R_{\gamma, z} b}}{w !} \\
& -\sum_{s=w}^{n-1} \frac{R_{\gamma, z}^{s}(-b)^{s-w}}{(s-w) !} \sum_{m=s+1}^{n} \frac{B_{z m}}{R_{\gamma, z}^{m}} \frac{E_{2, i+1} e^{R_{\gamma, z} b}}{w !}-\sum_{s=w-1}^{n-1} \frac{R_{\gamma, z}^{s}(-b)^{s-w+1}}{(s-w+1) !} \sum_{m=s+1}^{n} \frac{B_{z m}}{R_{\gamma, z}^{m}} \frac{E_{1, i+1} e^{R_{\gamma, z} b}}{(w-1) !} \\
& +\sum_{m=1 \vee(w-((j-i) n-1))}^{n} \frac{B_{z m}}{(m-1) !} \sum_{k=0 \vee(w-m)}^{(j-i) n-1} \sum_{p=0}^{k \wedge w} \frac{E_{k+3, i+1, z}\left(\begin{array}{c}
k \\
p
\end{array}\right)(-1)^{k-p}\left(\begin{array}{c}
m+k-p \\
w-p
\end{array}\right)(-b)^{m+k-w}}{m+k-p} \\
& +\sum_{m=1}^{n} \sum_{l=1, l \neq z}^{r} \frac{B_{l m}}{(m-1) !} \sum_{k=w}^{(j-i) n-1} \frac{E_{k+3, i+1, z}\left(\begin{array}{l}
k \\
w
\end{array}\right)(-1)^{k-w}(m+k-w-1) !}{\left(R_{\gamma, l}-R_{\gamma, z}\right)^{m+k-w}} \\
& +\sum_{l=1, l \neq z}^{r} e^{-R_{\gamma, l} b} \sum_{m=1 \vee(w-((j-i) n-2))}^{n} \frac{B_{z m}}{(m-1) !} \sum_{k=0 \vee(w-m+1)}^{(j-i) n-1} E_{k+3, i+1, l} \sum_{p=0}^{k \wedge w}\left(\begin{array}{l}
k \\
p
\end{array}\right)(-1)^{k-p+1}(m+k-p-1) ! \\
& \times \sum_{s=0 \vee(w-p)}^{m+k-p-1} \frac{\left(\begin{array}{c}
s \\
w-p
\end{array}\right)(-b)^{s-w+p}}{s !\left(R_{\gamma, z}-R_{\gamma, l}\right)^{m+k-p-s}} e^{R_{\gamma, z} b} \\
& +\sum_{s=w}^{n-1} \frac{(-b)^{s}}{(s-w) !} \sum_{m=s+1}^{n} B_{z m} \sum_{k=1}^{j n(r+1)} \frac{C_{k, i+1} e^{\alpha_{k} b}}{\left(R_{\gamma, z}+\alpha_{k}\right)^{m-s}} \frac{(-b)^{-w} e^{R_{\gamma, z} b}}{w !}, \quad z=1,2, \ldots, r ; \quad w=1,2, \ldots, n-1,
\end{aligned}
$$




$$
\begin{aligned}
& E_{w+3, i, z}=\sum_{m=1}^{n} \frac{B_{m}^{*}}{(m-1) !} \sum_{k=w}^{(j-i) n-1} \frac{E_{k+3, i+1, z}(m+k-w-1) !}{\left(\rho_{\gamma}+R_{\gamma, z}\right)^{m+k-w}} \\
& +\sum_{m=1 \vee(w-((j-i) n-1))}^{n} \frac{B_{z m}}{(m-1) !} \sum_{k=0 \vee(w-m)}^{(j-i) n-1} \sum_{p=0}^{k \wedge w} \frac{E_{k+3, i+1, z}\left(\begin{array}{l}
k \\
p
\end{array}\right)(-1)^{k-p}\left(\begin{array}{c}
m+k-p \\
w-p
\end{array}\right)(-b)^{m+k-w}}{m+k-p} \\
& +\sum_{m=1}^{n} \sum_{l=1, l \neq z}^{r} \frac{B_{l m}}{(m-1) !} \sum_{k=w}^{(j-i) n-1} \frac{E_{k+3, i+1, z}\left(\begin{array}{l}
k \\
w
\end{array}\right)(-1)^{k-w}(m+k-w-1) !}{\left(R_{\gamma, l}-R_{\gamma, z}\right)^{m+k-w}} \\
& +\sum_{l=1, l \neq z}^{r} e^{-R_{\gamma, l} b} \sum_{m=1 \vee(w-((j-i) n-2))}^{n} \frac{B_{z m}}{(m-1) !} \sum_{k=0 \vee(w-m+1)}^{(j-i) n-1} E_{k+3, i+1, l} \sum_{p=0}^{k \wedge w}\left(\begin{array}{l}
k \\
p
\end{array}\right)(-1)^{k-p+1}(m+k-p-1) ! \\
& \times \sum_{s=0 \vee(w-p)}^{m+k-p-1} \frac{\left(\begin{array}{c}
s \\
w-p
\end{array}\right)(-b)^{s-w+p}}{s !\left(R_{\gamma, z}-R_{\gamma, l}\right)^{m+k-p-s}} e^{R_{\gamma, z} b}, \quad z=1,2, \ldots, r ; \quad w=n, n+1, \ldots,(j-i) n-1, \\
& E_{w+3, i, z}=\sum_{m=1 \vee(w-((j-i) n-1))}^{n} \frac{B_{z m}}{(m-1) !} \sum_{k=0 \vee(w-m)}^{(j-i) n-1} \sum_{p=0}^{k \wedge w} \frac{E_{k+3, i+1, z}\left(\begin{array}{l}
k \\
p
\end{array}\right)(-1)^{k-p}\left(\begin{array}{c}
m+k-p \\
w-p
\end{array}\right)(-b)^{m+k-w}}{m+k-p} \\
& +\sum_{l=1, l \neq z}^{r} e^{-R_{\gamma, l} b} \sum_{m=1 \vee(w-((j-i) n-2))}^{n} \frac{B_{z m}}{(m-1) !} \sum_{k=0 \vee(w-m+1)}^{(j-i) n-1} E_{k+3, i+1, l} \sum_{p=0}^{k \wedge w}\left(\begin{array}{l}
k \\
p
\end{array}\right)(-1)^{k-p+1}(m+k-p-1) ! \\
& \times \sum_{s=0 \vee(w-p)}^{m+k-p-1} \frac{\left(\begin{array}{c}
s \\
w-p
\end{array}\right)(-b)^{s-w+p}}{s !\left(R_{\gamma, z}-R_{\gamma, l}\right)^{m+k-p-s}} e^{R_{\gamma, z} b}, \quad z=1,2, \ldots, r ; \quad w=(j-i) n,(j-i) n+1, \ldots,(j-i+1) n-2, \\
& E_{(j-i+1) n+2, i, z}=\frac{B_{z n}}{(n-1) !} \sum_{p=0}^{(j-i) n-1} \frac{E_{(j-i) n+2, i+1, z}\left(\begin{array}{c}
(j-i) n-1 \\
p
\end{array}\right)(-1)^{(j-i) n-1-p}}{(j-i+1) n-1-p}, \quad z=1,2, \ldots, r .
\end{aligned}
$$

Using (3.14), (3.15), (3.18) and (3.19), the general expressions of $E_{1, i}$ and $E_{2, i}$ are given by, for $i=$ $2,3, \ldots, j$,

$$
\begin{aligned}
E_{1, i}= & \left(\frac{\gamma}{\gamma+\delta}\right)^{n(j-i+1)}, \\
E_{2, i}= & \left(\frac{\gamma}{\gamma+\delta}\right)^{n(j-i+1)}\left(V_{1}(b ; b)-b\right) \\
& +(j-i+1)\left(\sum_{m=1}^{n} \frac{B_{m}^{*}}{\rho_{\gamma}^{m}}+\sum_{z=1}^{r} \sum_{m=1}^{n} \frac{B_{z m}}{R_{\gamma, z}^{m}}\right)^{j-i}\left(\sum_{m=1}^{n} \frac{B_{m}^{*} m}{\rho_{\gamma}^{m+1}}-\sum_{z=1}^{r} \sum_{m=1}^{n} \frac{B_{z m} m}{R_{\gamma, z}^{m+1}}\right) .
\end{aligned}
$$

Similar to Section 2.2, the expected discounted dividends paid until ruin can be fully characterized by the above equations. For easy reference, the entire computational procedure is highlighted as follows.

1. Under the distributional assumption (3.1) on the claims, solve (3.3) to obtain $\rho_{\gamma}>0$ and $\left\{-R_{\gamma, i}\right\}_{i=1}^{r}$ which have negative real parts.

2. Determine (as in Albrecher et al. (2011b)) $B_{j}^{*}$ 's and $B_{i j}$ 's using (3.4) and (3.5), or equivalently, via the partial fractions expansion

$$
\left(\frac{\gamma}{c}\right)^{n} \frac{\left[Q_{1, r}(s)\right]^{n}}{\left(\rho_{\gamma}-s\right)^{n} \prod_{i=1}^{r}\left(s+R_{\gamma, i}\right)^{n}}=\sum_{j=1}^{n} \frac{B_{j}^{*}}{\left(\rho_{\gamma}-s\right)^{j}}+\sum_{i=1}^{r} \sum_{j=1}^{n} \frac{B_{i j}}{\left(s+R_{\gamma, i}\right)^{j}} .
$$

3. For each fixed $l=0,1, \ldots, j-1$, solve the $n(r+1)$ roots of $(2.40)$ for $\alpha_{k}$, where $Z(\cdot)$ is defined in (3.10). This results in a total of $j n(r+1)$ values denoted by $\left\{\alpha_{k}\right\}_{k=1}^{j n(r+1)}$. 
4. With $E_{1, i}$ explicitly given in (3.25), use (3.6) at $x=b$ along with (2.53), (3.16), (3.17), (3.20)(3.24) and (3.26) to express $\left\{C_{k, i}\right\}_{k=1}^{j n(r+1)}, E_{2, i}$ and $\left\{E_{k+3, i, l}\right\}_{k=0}^{(j-i+1) n-1}$ (for $l=1,2, \ldots, r$ and $i=2,3, \ldots, j)$ in terms of $\left\{C_{k, 1}\right\}_{k=1}^{j n(r+1)}$.

5. Solve the system of $j n(r+1)$ linear equations which comprise $j n r$ equations from $(3.11),(j-1) n$ from (3.12) and $n$ from (3.13) to obtain $\left\{C_{k, 1}\right\}_{k=1}^{j n(r+1)}$.

6. Use the resulting values of $\left\{C_{k, 1}\right\}_{k=1}^{j n(r+1)}$ to compute the coefficients in Step 4 above.

7. The expected discounted dividends before ruin can finally be computed by means of (3.6)-(3.9).

Remark 3.1 From (3.8), (3.9) and (3.25), one can easily see that, for $i=2,3, \ldots, j$,

$$
V_{i, U}(x ; b) \sim\left(\frac{\gamma}{\gamma+\delta}\right)^{n(j-i+1)} x, \quad x \rightarrow \infty .
$$

The above asymptotic formula has an intuitive explanation. By recalling that the subscript $i$ means that there are $j-i+1$ observation periods (each of which is $\operatorname{Erlang}(n)$ distributed) before the next dividend-decision time, we note that $[\gamma /(\gamma+\delta)]^{n(j-i+1)}$ is precisely the expected discounted value (under force of interest $\delta$ ) of one dollar paid at the next dividend-decision time. When the initial surplus $x$ is very large, it is highly likely that the first dividend payment will be paid at the next dividend-decision time and the size of the payment is essentially $x$ dollars. See also Avanzi et al. (2013, Remark 3.2) for a similar probabilistic interpretation.

Remark 3.2 When reconciling our results with Albrecher et al. (2011b) who considered the dividend moments under the case $j=1$, a typo is found in the denominator of their equation (48). In their notations, the correct expression is given by

$$
\sum_{p=1}^{n(r+1)} A_{p, m} \sum_{j=i}^{n} \frac{B_{k j, m}}{\left(R_{\gamma, k, m}+\alpha_{p, m}\right)^{j-i+1}}=0, \quad k=1,2, \ldots, r ; \quad i=1,2, \ldots, n .
$$

Then, our (3.13), (2.40) (with extended domain $k=1,2, \ldots, j n(r+1))$ and (3.11) respectively correspond to equations (46)-(48) in Albrecher et al. (2011b). There is also a similar typo in their equation (57), in which the exponent in the denominator should be $j-i+1$ instead of $j$.

\section{Numerical illustrations}

In this section, the theoretical results in Section 3 are applied to generate numerical examples. In particular, given that solvency is checked at the epochs $\left\{L_{k}\right\}_{k=0}^{\infty}$, we are interested in choosing the value of $j$ for the dividend-decision times $\left\{L_{j k}\right\}_{k=0}^{\infty}$ to maximize the expected discounted dividends paid until ruin. The (joint) optimality of $b$ and $j$ will be studied. As discussed in Section 1, optimization with respect to $j$ helps answer the following question: if the insurer balances its books monthly and a barrier strategy is implemented, then is it more optimal to make dividend decisions quarterly, semi-annually or annually? Like the usual optimal barrier that maximizes dividends, deciding on the optimal $j$ is not a trivial problem. Two opposite effects are in place when one increases or decreases $j$ for a fixed barrier level $b$. A small (large) $j$ is expected to result in more (less) frequent dividend decisions and hence earlier (later) dividend payments, which is favourable (unfavourable) in the presence of discounting. However, 
such a higher (lower) dividend payout at an early time will lead to earlier (later) ruin time and hence less (more) future dividends.

In calculating all the dividend values, the steps outlined near the end of Section 3 are adopted. First, we follow closely the parameters and claim distributions as in Albrecher et al. (2011b, Table 1-3). Specifically, it is always assumed that the premium rate is $c=1.5$, the Poisson claim arrival rate is $\lambda=1$, and the force of interest is $\delta=0.005$. In addition, to illustrate the Erlangization technique, we consider Erlang $(n)$ inter-observation time $T$ up to $n=6$ while the mean is fixed to be $\mathbb{E}[T]=2.5$. The three claim distributions used include (i) a sum of two exponentials; (ii) exponential; and (iii) a mixture of two exponentials. Their respective densities are given at the bottom of Tables 1-3. While they all have the same mean $\mathbb{E}[Y]=1$, their variances are respectively $0.56,1$ and 2 . In producing Tables 1-3, for each fixed $n, j, i$ (where $i=1,2, \ldots, j$ ) and initial surplus $x$, we start by computing numerically the optimal barrier level $b^{*}$ which maximizes the dividend function $V_{i}(x ; b)$ with respect to $b$. It is found that the value of $b^{*}$ is independent of $x$ and $i$ for each fixed pair of $n$ and $j$. The independence of $b^{*}$ on $x$ is well known in the classical model under the traditional barrier strategy (e.g. Gerber et al. (2006)). In addition, the index $i$ (which indicates that the time until the first dividend decision is distributed as $\sum_{k=1}^{j-i+1} T_{k}$ ) resembles the initial environmental state in Markov-modulated models, and the independence of $b^{*}$ on the initial environmental state in Markovian models can be found in e.g. Zhang and Cheung (2014a, Section 5.2). The resulting optimal dividend values $V_{i}\left(x ; b^{*}\right)$ are summarized in Tables $1-3$ for $j=1,2,3$ and various values of initial surplus $x$. Although Tables $1-3$ only show the dividend values in the lower layer $0 \leq x \leq b^{*}$, we have indeed verified numerically that for each fixed pair of $n$ and $j$ it is the same $b^{*}$ that maximizes $V_{i, U}(x ; b)$ for all $i=2,3, \ldots, j$ and $x>b^{*}$. For the sake of brevity these results are not presented here.

From each of Tables 1-3, for fixed $n$ it can be observed that $b^{*}$ decreases as $j$ increases. Intuitively, a higher $j$ means longer intervals between dividend-decisions, and hence a lower $b^{*}$ is required to maximize dividends (otherwise dividends will hardly be paid). Note that our values for the case $j=1$ in each table are consistent with those in Albrecher et al. (2011b). For fixed pair of $n$ and $j$, the dividend value $V_{i}\left(x ; b^{*}\right)$ is always increasing in $x$ for $0 \leq x \leq b^{*}$ as usual. Moreover, $V_{i}\left(x ; b^{*}\right)$ appears to be quite insensitive to the value of $i$. Within each table, it can be observed that the dividend values do not change much as $n$ increases further from moderate values of $n$ (say $n=4$ or $n=5$ ), illustrating the effect of Erlangization. Similar to Albrecher et al. (2011b), when one compares the values across Tables 1-3, as the variance of the claim severity increases (i.e. the surplus process gets riskier), the optimal barrier $b^{*}$ appears to increase (so that the insurer can retain more surplus to avoid early ruin and more future dividends can be paid) while $V_{i}\left(x ; b^{*}\right)$ (for $\left.x=0,5,10\right)$ decreases.

In addition, the behaviour of $V_{i}^{\prime}(x ; b)$ against $x$ for $i=1,2,3$ and $b=5, b^{*}, 20$ is depicted graphically in Figure 2 using the parameters as in Table 1 with $n=1$ and $j=3$. In this case, one has $b^{*}=12.09$. In Figure 2, for $i=2,3$, as $x$ increases one observes that $V_{i}^{\prime}(x ; b)$ converges to a value slightly less than 1. This can be attributed to Remark 3.1 so that $\lim _{x \rightarrow \infty} V_{i}^{\prime}(x ; b)=[\gamma /(\gamma+\delta)]^{n(j-i+1)}$ which equals 0.975 when $i=2$ and 0.988 when $i=3$. Moreover, it can be seen from Figure 2 that $V_{i}^{\prime}\left(x ; b^{*}\right)=1$ at $x=b^{*}=12.09$ for $i=1,2,3$. Indeed, for the parameter set and claims distributions that are used in Tables 1-3, we have numerically verified that

$$
V_{i}^{\prime}\left(b^{*} ; b^{*}\right)=1, \quad i=1,2,3 .
$$

As a result, $b^{*}$ can be interpreted as the optimal financial capital (see Albrecher et al. (2011c, Section 8, 
Remark (ii))). Another consequence of (4.1) is

$$
\left.\frac{d}{d b} V_{i}(b ; b)\right|_{b=b^{*}}=1,, \quad i=1,2,3 .
$$

See e.g. Avanzi et al. (2007, Section 5 and 2013, Section 4.3). Although so far we have only considered $V(x ; b)=V_{1}(x ; b)$ for $0 \leq x \leq b$, with $L_{0}$ being a dividend decision time one can readily extend the domain such that $V(x ; b)=x-b+V(b ; b)$ for $x>b$. Therefore, (4.2) at $i=1$ implies

$$
\left.\frac{\partial}{\partial b} V(x ; b)\right|_{b=b^{*}}=-1+\left.\frac{d}{d b} V(b ; b)\right|_{b=b^{*}}=0, \quad x>b^{*},
$$

\begin{tabular}{|c|c|c|c|c|c|c|c|c|c|c|c|c|c|c|c|c|c|c|}
\hline \multirow{3}{*}{$\begin{array}{c}\text { Sum Exp } \\
i\end{array}$} & \multicolumn{6}{|c|}{$n=1$} & \multicolumn{6}{|c|}{$n=2$} & \multicolumn{6}{|c|}{$n=3$} \\
\hline & $|j=1|$ & \multicolumn{2}{|c|}{$j=2$} & \multicolumn{3}{|c|}{$j=3$} & \multirow{2}{*}{$\frac{|j=1|}{1}$} & \multicolumn{2}{|c|}{$j=2$} & \multicolumn{3}{|c|}{$j=3$} & \multirow{2}{*}{$\frac{|j=1|}{\mid 1}$} & \multicolumn{2}{|c|}{$j=2$} & \multicolumn{3}{|c|}{$j=3$} \\
\hline & 1 & 1 & 2 & 1 & 2 & 3 & & 1 & 2 & 1 & 2 & 3 & & 1 & 2 & 1 & 2 & 3 \\
\hline$b^{*}$ & 12.98 & 12.49 & 12.49 & 12.09 & 12.09 & 12.09 & 13.27 & 12.73 & 12.73 & 12.31 & 12.31 & 12.31 & 13.37 & 12.81 & 12.81 & 12.38 & 12.38 & 12.38 \\
\hline$V_{i}\left(0 ; b^{*}\right)$ & 55.47 & 55.28 & 55.28 & 55.08 & 55.08 & 55.08 & 55.27 & 55.10 & 55.10 & 54.91 & 54.91 & 54.91 & 55.34 & 55.17 & 55.17 & 54.98 & 54.98 & 54.98 \\
\hline$V_{i}\left(5 ; b^{*}\right)$ & 86.83 & 86.54 & 86.54 & 86.23 & 86.23 & 86.23 & 86.67 & 86.40 & 86.40 & 86.11 & 86.11 & 86.11 & 86.61 & 86.34 & 86.34 & 86.05 & 86.05 & 86.05 \\
\hline$V_{i}\left(10 ; b^{*}\right)$ & 94.27 & 93.96 & 93.96 & 93.62 & 93.62 & 93.62 & 94.30 & 94.00 & 94.00 & 93.68 & 93.68 & 93.68 & 94.30 & 94.01 & 94.01 & 93.70 & 93.70 & 93.69 \\
\hline \multirow[t]{3}{*}{$V_{i}\left(b^{*} ; b^{*}\right)$} & |97.34 & 96.53 & 96.53 & 95.78 & 95.79 & 95.79 & 97.68 & 96.83 & 96.84 & 96.07 & |96.08 & 96.08 & $|97.79|$ & 96.93 & 96.94 & 96.17 & 96.18 & 96 \\
\hline & \multicolumn{6}{|c|}{$n=4$} & \multicolumn{6}{|c|}{$n=5$} & \multicolumn{6}{|c|}{$n=6$} \\
\hline & $|j=1|$ & \multicolumn{2}{|c|}{$j=2$} & \multicolumn{3}{|c|}{$j=3$} & $|j=1|$ & \multicolumn{2}{|c|}{$j=2$} & \multicolumn{3}{|c|}{$j=3$} & $|j=1|$ & \multicolumn{2}{|c|}{$j=2$} & \multicolumn{3}{|c|}{$j=3$} \\
\hline$i$ & 1 & 1 & 2 & 1 & 2 & 3 & 1 & 1 & 2 & 1 & 2 & 3 & 1 & 1 & 2 & 1 & 2 & 3 \\
\hline$b^{*}$ & 13.42 & 12.86 & 12.86 & 12.42 & 12.42 & 12.42 & 13.45 & 12.88 & 12.88 & 12.44 & 12.44 & 12.44 & 13.47 & 12.90 & 12.90 & 12.46 & 12.46 & 12.46 \\
\hline$V_{i}\left(0 ; b^{\prime}\right.$ & 55.42 & 55.25 & 55.25 & 55.06 & 55.06 & 55.06 & 55.48 & 55.31 & 55.31 & 55.13 & 55.13 & 55.13 & 55.52 & 55.36 & 55.36 & 55.17 & 55.17 & 55.17 \\
\hline$V_{i}\left(5 ; b^{*}\right)$ & 86.57 & 86.31 & 86.31 & 86.02 & 86.02 & 86.02 & 86.55 & 86.29 & 86.29 & 86.00 & 86.00 & 86.00 & 86.53 & 86.28 & 86.28 & 85.99 & 85.99 & 85. \\
\hline$V_{i}\left(10 ; b^{*}\right)$ & 94.30 & 94.02 & 94.02 & 93.70 & 93.70 & 93.70 & 94.30 & 94.02 & 94.02 & 93.71 & 93.71 & 93.70 & 94.30 & 94.02 & 94.02 & 93.71 & 93.71 & 93.71 \\
\hline , & 97.84 & 96.98 & 96.99 & 96.21 & 96.22 & 96.22 & 97.88 & 97.01 & 97.02 & 96.24 & 96.25 & 96.25 & 97.90 & 97.03 & 97.04 & 96.26 & 96.27 & 96.27 \\
\hline
\end{tabular}

i.e. the same $b^{*}$ maximizes $V(x ; b)$ for all $x \geq 0$.

\begin{tabular}{|c|c|c|c|c|c|c|c|c|c|c|c|c|c|c|c|c|c|c|}
\hline \multirow{4}{*}{$\begin{array}{c}\text { Exp } \\
\\
i \\
b^{*}\end{array}$} & \multicolumn{6}{|c|}{$n=1$} & \multicolumn{6}{|c|}{$n=2$} & \multicolumn{6}{|c|}{$n=3$} \\
\hline & $|j=1|$ & \multicolumn{2}{|c|}{$j=2$} & \multicolumn{3}{|c|}{$j=3$} & \multirow{2}{*}{$\frac{|j=1|}{1}$} & \multicolumn{2}{|c|}{$j=2$} & \multicolumn{3}{|c|}{$j=3$} & \multirow{2}{*}{$\frac{|j=1|}{1}$} & \multicolumn{2}{|c|}{$j=2$} & \multicolumn{3}{|c|}{$j=3$} \\
\hline & 1 & 1 & 2 & 1 & 2 & 3 & & 1 & 2 & 1 & 2 & 3 & & 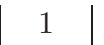 & 2 & 1 & 2 & 3 \\
\hline & 15.93 & 15.37 & 15.37 & 14.90 & 14.90 & 14.90 & 16.28 & 15.66 & 15.66 & 15.16 & 15.16 & 15.16 & 16.40 & 15.76 & 15.76 & 15.25 & 15.25 & 15.25 \\
\hline$V_{i}\left(0 ; b^{*}\right)$ & 51.66 & 51.50 & 51.50 & 51.33 & 51.33 & 51.33 & 51.18 & 51.04 & 51.04 & 50.88 & 50.88 & 50.88 & 51.10 & $|50.97|$ & 50.97 & 50.81 & 50.81 & 50.81 \\
\hline & 81.48 & 81.24 & 81.24 & 80.97 & 80.97 & 80.97 & 81.17 & 80.94 & 80.94 & 80.69 & 80.69 & 80.69 & 81.05 & 80.83 & 80.83 & 80.58 & 80.58 & 80.58 \\
\hline$V_{i}\left(10 ; b^{*}\right)$ & 90.56 & 90.29 & 90.29 & 89.99 & 89.99 & 89.99 & 90.50 & 90.25 & 90.25 & 89.97 & 89.97 & 89.97 & 90.48 & 90.23 & 90.23 & 89.95 & 89.95 & 89.95 \\
\hline \multirow{3}{*}{$V_{i}\left(b^{*} ; b^{*}\right)$} & $|96.95|$ & $|96.10|$ & 96.10 & 95.31 & |95.32 | & 95.32 & $|97.30|$ & |96.41| & 96.41 & $|95.61|$ & 95.61 & 95.61 & $|97.42|$ & $|96.51|$ & 96.52 & 95.70 & 95.71 & 95. \\
\hline & \multicolumn{6}{|c|}{$n=4$} & \multicolumn{6}{|c|}{$n=5$} & \multicolumn{6}{|c|}{$n=6$} \\
\hline & $|j=1|$ & \multicolumn{2}{|c|}{$j=2$} & \multicolumn{3}{|c|}{$j=3$} & $|j=1|$ & \multicolumn{2}{|c|}{$j=2$} & \multicolumn{3}{|c|}{$j=3$} & $|j=1|$ & \multicolumn{2}{|c|}{$j=2$} & \multicolumn{3}{|c|}{$j=3$} \\
\hline$i$ & 1 & 1 & 2 & 1 & 2 & 3 & 1 & 1 & 2 & 1 & 2 & 3 & 1 & 1 & 2 & 1 & 2 & 3 \\
\hline 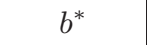 & 16.46 & 15.81 & 15.81 & 15.30 & 15.30 & 15.30 & 16.50 & 15.84 & 15.84 & 15.33 & 15.33 & 15.33 & 16.53 & $\mid 15.86$ & 15.86 & 15.35 & 15.35 & 15.35 \\
\hline$V_{t}$ & 51.09 & 50.96 & 50.96 & 50.80 & 50.80 & 50.80 & 51.10 & 50.96 & 50.96 & 50.81 & 50.81 & 50.81 & 51.11 & $\mid 50.97$ & 50.97 & 50.82 & 50.82 & 50. \\
\hline$V_{i}\left(5 ; b^{*}\right)$ & 80.98 & 80.77 & 80.77 & 80.52 & 80.52 & 80.52 & 80.94 & 80.73 & 80.73 & 80.48 & 80.48 & 80.48 & 80.91 & 80.70 & 80.70 & 80.46 & 80.46 & 80.46 \\
\hline$V_{i}\left(10 ; b^{*}\right)$ & 90.46 & 90.22 & 90.22 & 89.94 & 89.94 & 89.94 & 90.45 & 90.21 & 90.21 & 89.94 & 89.94 & 89.94 & 90.45 & 90.21 & 90.21 & 89.94 & 89.93 & 89.94 \\
\hline$V_{i}\left(b^{*} ; b^{*}\right)$ & 97.48 & 96.56 & 96.57 & 95.75 & 95.76 & 95.76 & 97.52 & 96.60 & 96.60 & 95.78 & 95.79 & 95.79 & 97.54 & 96.62 & 96.62 & 95.80 & 95.81 & 95.81 \\
\hline
\end{tabular}

Table 1: $c=1.5, \lambda=1, \delta=0.005, f_{Y}(y)=3 e^{-1.5 y}-3 e^{-3 y}, T \sim \operatorname{Erlang}(n)$ with $\mathbb{E}[T]=2.5$

Table 2: $c=1.5, \lambda=1, \delta=0.005, f_{Y}(y)=e^{-y}, T \sim \operatorname{Erlang}(n)$ with $\mathbb{E}[T]=2.5$ 


\begin{tabular}{|c|c|c|c|c|c|c|c|c|c|c|c|c|c|c|c|c|c|c|}
\hline \multirow{3}{*}{$\begin{array}{c}\text { Mixed Exp } \\
i\end{array}$} & \multicolumn{6}{|c|}{$n=1$} & \multicolumn{6}{|c|}{$n=2$} & \multicolumn{6}{|c|}{$n=3$} \\
\hline & $|j=1|$ & \multicolumn{2}{|c|}{$j=2$} & \multicolumn{3}{|c|}{$j=3$} & $|j=1|$ & \multicolumn{2}{|c|}{$j=2$} & \multicolumn{3}{|c|}{$j=3$} & $|j=1|$ & \multicolumn{2}{|c|}{$j=2$} & \multicolumn{3}{|c|}{$j=3$} \\
\hline & 1 & 1 & 2 & 1 & 2 & 3 & 1 & 1 & 2 & 1 & 2 & 3 & 1 & 1 & 2 & 1 & 2 & 3 \\
\hline$b^{*}$ & 21.87 & 21.18 & 21.18 & 20.58 & 20.58 & 20.58 & 22.35 & 21.58 & 21.58 & 20.95 & 20.95 & 20.95 & 22.51 & 21.72 & 21.72 & 21.08 & 21.08 & 21.08 \\
\hline$V_{i}\left(0 ; b^{*}\right)$ & 46.22 & 46.11 & 46.11 & 45.99 & 45.99 & 45.99 & 45.58 & 45.48 & 45.48 & 45.36 & 45.36 & 45.36 & 45.40 & 45.30 & 45.30 & 45.19 & 45.19 & 45.19 \\
\hline$V_{i}\left(5 ; b^{*}\right)$ & 71.03 & 70.86 & 70.86 & 70.67 & 70.67 & 70.67 & 70.48 & 70.32 & 70.32 & 70.14 & 70.14 & 70.14 & 70.26 & 70.12 & 70.12 & 69.94 & 69.94 & 69.94 \\
\hline$V_{i}\left(10 ; b^{*}\right)$ & 82.03 & 81.83 & 81.83 & 81.61 & 81.61 & 81.61 & 81.78 & 81.60 & 81.60 & 81.39 & 81.39 & 81.39 & 81.68 & 81.51 & 81.51 & 81.30 & 81.30 & 81.30 \\
\hline \multirow[t]{3}{*}{$V_{i}\left(b^{*} ; b^{*}\right)$} & 95.94 & 95.01 & 95.01 & 94.16 & 94.16 & 94.16 & 96.32 & 95.34 & 95.35 & 94.46 & 94.47 & 94.47 & 96.45 & 95.45 & 95.46 & 94.57 & 94.57 & 94.57 \\
\hline & \multicolumn{6}{|c|}{$n=4$} & \multicolumn{6}{|c|}{$n=5$} & \multicolumn{6}{|c|}{$n=6$} \\
\hline & $|j=1|$ & \multicolumn{2}{|c|}{$j=2$} & \multicolumn{3}{|c|}{$j=3$} & $|j=1|$ & \multicolumn{2}{|c|}{$j=2$} & \multicolumn{3}{|c|}{$j=3$} & $|j=1|$ & \multicolumn{2}{|c|}{$j=2$} & \multicolumn{3}{|c|}{$j=3$} \\
\hline$i$ & 1 & 1 & 2 & 1 & 2 & 3 & 1 & 1 & 2 & 1 & 2 & 3 & 1 & 1 & 2 & 1 & 2 & 3 \\
\hline$b^{*}$ & 22.60 & 21.79 & 21.79 & 21.14 & 21.14 & 21.14 & 22.65 & 21.84 & 21.84 & 21.18 & 21.18 & 21.18 & 22.69 & 21.87 & 21.87 & 21.21 & 21.21 & 21.21 \\
\hline$V_{i}\left(0 ; b^{*}\right)$ & 45.31 & 45.22 & 45.22 & 45.11 & 45.11 & 45.11 & 45.27 & 45.18 & 45.18 & 45.07 & 45.07 & 45.07 & 45.24 & 45.15 & 45.15 & 45.04 & 45.04 & 45.04 \\
\hline$V_{i}\left(5 ; b^{*}\right)$ & 70.15 & 70.01 & 70.01 & 69.84 & 69.84 & 69.84 & 70.09 & 69.95 & 69.95 & 69.78 & 69.78 & 69.78 & 70.04 & 69.90 & 69.90 & 69.73 & 69.73 & 69.73 \\
\hline$V_{i}\left(10 ; b^{*}\right)$ & 81.62 & 81.46 & 81.46 & 81.26 & 81.26 & 81.26 & 81.59 & 81.43 & 81.43 & 81.23 & 81.23 & 81.23 & 81.57 & 81.41 & 81.41 & 81.21 & 81.21 & 81.21 \\
\hline$V_{i}\left(b^{*} ; b^{*}\right)$ & 96.52 & 95.51 & 95.51 & 94.62 & 94.63 & 94.62 & 96.56 & 95.54 & 95.55 & 94.65 & 94.66 & 94.65 & 96.58 & 95.57 & 95.57 & 94.67 & 94.68 & 94.67 \\
\hline
\end{tabular}

Table 3: $c=1.5, \lambda=1, \delta=0.005, f_{Y}(y)=(1 / 6) e^{-0.5 y}+(4 / 3) e^{-2 y}, T \sim$ Erlang $(n)$ with $\mathbb{E}[T]=2.5$
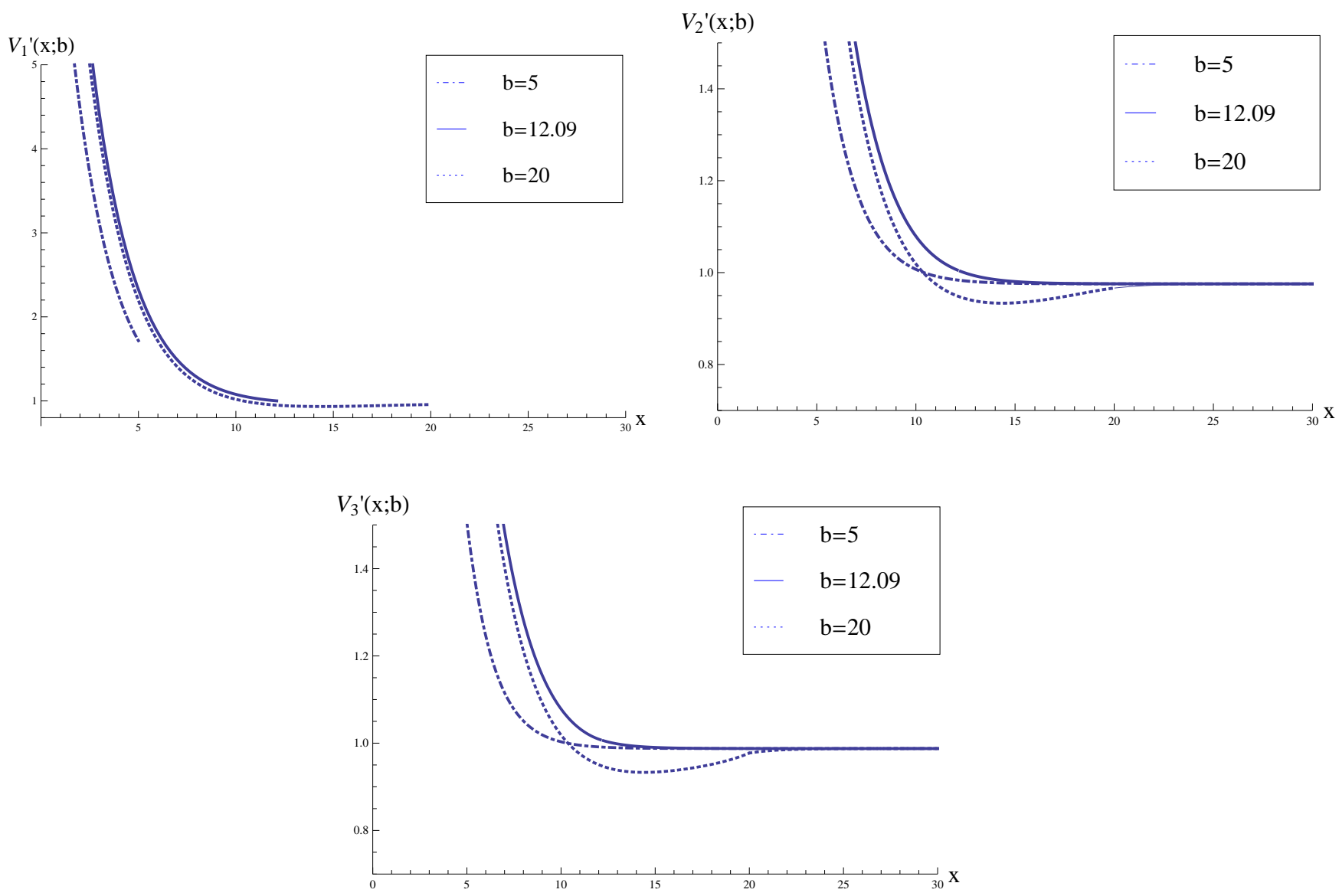

Figure 2: $V_{1}^{\prime}(x ; b), V_{2}^{\prime}(x ; b)$ and $V_{3}^{\prime}(x ; b)$ for $b=5$ (dot-dashed line), $b=b^{*}=12.09$ (solid line) and $b=20$ (dotted line) with parameters $c=1.5, \lambda=1, \delta=0.005, f_{Y}(y)=3 e^{-1.5 y}-3 e^{-3 y}, T \sim \operatorname{Erlang}(1)$ with $\mathbb{E}[T]=2.5$ and $j=3$ 
Concerning dividend maximization, suppose that the pair $(b, j)$ can be freely selected in order to maximize $V(x ; b)$ at time 0 . This can be regarded as a two-step procedure. First, an appropriate value of $n$ is chosen, and for each fixed $j$ one determines $b^{*}=b^{*}(j)$ (here we emphasize the dependence on $j$ ) that maximizes $V(x ; b)$ with respect to $b$. The second step is to select $j^{*}$ to maximize $V\left(x ; b^{*}(j)\right)$ with respect to $j$, and therefore the optimal pair of $(b, j)$ is given by $\left(b^{*}\left(j^{*}\right), j^{*}\right)$. By examining the columns for the case $i=1$ in Tables $1-3$, it is easy to see that $\left(b^{*}\left(j^{*}\right), j^{*}\right)=\left(b^{*}(1), 1\right)$ for all $n=1,2, \ldots, 6$. In other words, under our parameter set and claim distributions, it is optimal to make dividend decisions at all of the observation time points if the barrier is chosen to be $b^{*}(1)$. Intuitively, the result $j^{*}=1$ should not be surprising at least for (mixed) exponential claim distributions where $f_{Y}(\cdot)$ is completely monotone. In this case, it is known from Loeffen (2008, Theorem 3) that the classical (continuous) barrier strategy is the dividend strategy (among all admissible strategies) that maximizes the expected discounted dividends (until the time of ruin in the traditional sense). Hence, when the insurer can choose how often to pay dividends in the present model to maximize dividends, it is natural to expect dividend decisions to be made as frequent as possible (i.e. $j^{*}=1$ ) and then the barrier level $b^{*}(1)$ will be adjusted accordingly.

However, it is possible that the insurer or the shareholders may not want to alter the dividend barrier that is already in place even it is not optimal. In such a case, the only free parameter for dividend maximization is the value of $j$. This leads to a different optimization problem of determining the optimal value of $j$, say $j^{*}(b)$, for a fixed barrier level $b$. This can be treated as the reverse case of first fixing $j$ and then finding the optimal value of $b$ as in Tables 1-3. In what follows, we consider a different set of parameters with premium rate $c=6$, Poisson rate $\lambda=15$ and force of interest $\delta=0.05$. For simplicity, it is assumed that the claims are exponential with mean $1 / \nu=1 / 3$ and inter-observation times are also exponential but with mean $1 / \gamma=1$. Figures 3 and 4 plot the dividend function $V_{1}(x ; b)$ for various values of $j$ when the barrier levels are $b=1$ and $b=5$ respectively. In Figure 3, it is clear that $j=9$ gives the highest value of $V_{1}(x ; 1)$ compared to $j=1,3,5,7,9,10$ for all $0 \leq x \leq 1$. We have verified that $j^{*}(1)=9$ but the cases $j=2,4,6,8$ are omitted from the figure for clarity of presentation. Intuitively, the barrier level $b=1$ is relatively small. This means that after each dividend decision time, the surplus process is fairly close to ruin as the surplus level cannot exceed 1 . If a small value of $j$ is applied, the surplus process is very likely to ruin early. Therefore, to maximize dividends when $b$ is low, it may be more advantageous to delay dividend decisions so that more dividends can be accumulated in the future, resulting in an optimal value $j^{*}(b)$ that is not necessarily 1 . (Recall that when we optimize with respect to both $j$ and $b$, the optimal value of $j$ is found to be 1 according to the discussion in the previous paragraph.) When we turn to Figure 4 where $b=5$, the optimal value of $j$ is given by $j^{*}(5)=1$ for all $0 \leq x \leq 5$. In this case, a higher barrier level means that the surplus process is not close to ruin even after a dividend decision, and hence distributing dividends at earlier times may give higher value of dividends.

\section{Acknowledgements}

The authors would like to thank the anonymous referee for helpful comments and suggestions which improved an earlier version of the paper. Eric Cheung gratefully acknowledges the support from the Research Grants Council of the Hong Kong Special Administrative Region (Project Number: HKU $701212 \mathrm{P})$. 


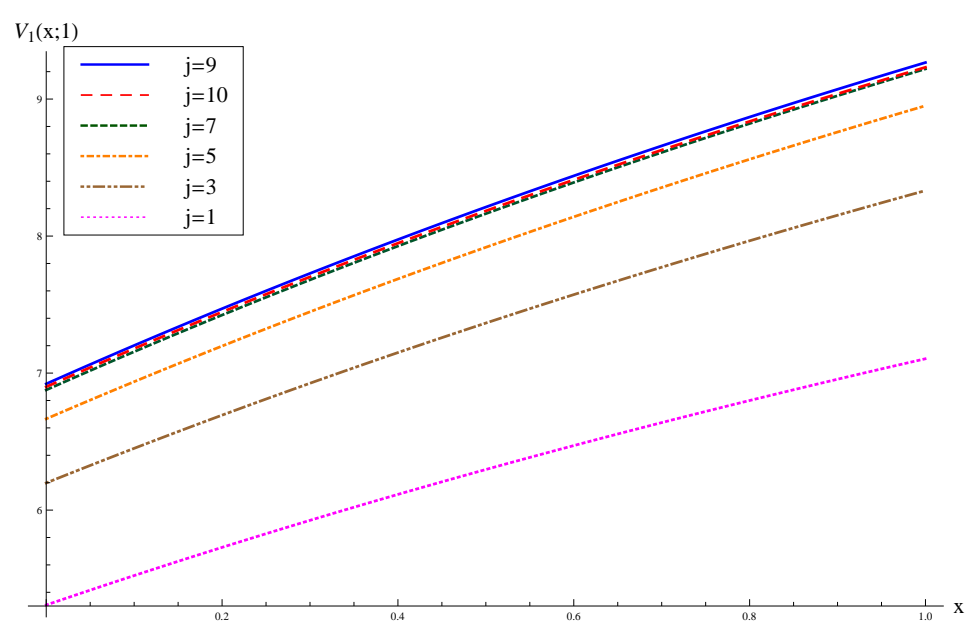

Figure 3: $V_{1}(x ; 1)$ for $j=1,3,5,7,9,10$ with parameters $c=$ $6, \nu=3, \lambda=15, \delta=0.05, \gamma=1, n=1$

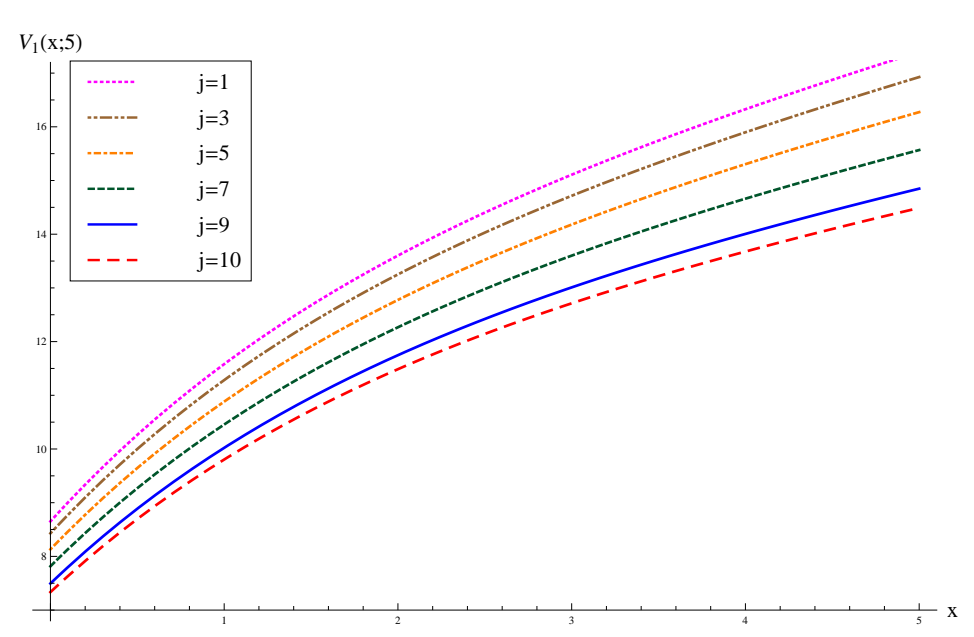

Figure 4: $V_{1}(x ; 5)$ for $j=1,3,5,7,9,10$ with parameters $c=$ $6, \nu=3, \lambda=15, \delta=0.05, \gamma=1, n=1$

\section{References}

[1] Albrecher, H., Bäuerle, N. And Thonhauser, S. 2011a. Optimal dividend-payout in random discrete time. Statistics and Risk Modeling 28(3): 251-276.

[2] Albrecher, H., Cheung, E.C.K. And Thonhauser, S. 2011b. Randomized observation periods for the compound Poisson risk model: Dividends. ASTIN Bulletin 41(2): 645-672. 
[3] Albrecher, H., Cheung, E.C.K. And Thonhauser, S. 2013. Randomized observation periods for the compound Poisson risk model: The discounted penalty function. Scandinavian Actuarial Journal 2013(6): 424-452.

[4] Albrecher, H., Gerber, H.U. and Shiu, E.S.W. 2011c. The optimal dividend barrier in the Gamma-Omega model. European Actuarial Journal 1(1): 43-55.

[5] Albrecher, H. And Ivanovs, J. 2013. A risk model with an observer in a Markov environment. Risks 1(3): 148-161.

[6] Albrecher, H., Ivanovs, J. and Zhou, X. 2014. Exit identities for Lévy processes observed at Poisson arrival times. Preprint.

[7] Albrecher, H. and Lautscham, V. 2013. From ruin to bankruptcy for compound Poisson surplus processes. ASTIN Bulletin 43(2): 213-243.

[8] Albrecher, H. and Thonhauser, S. 2009. Optimality results for dividend problems in insurance. Revista de la Real Academia de Ciencias Exactas, Fisicas y Naturales Serie A. Matematicas 103(2): 295-320.

[9] Asmussen, S. And Albrecher, H. 2010. Ruin Probabilities. Second Edition. New Jersey: World Scientific.

[10] Asmussen, S., Avram F. and Usabel, M. 2002. Erlangian approximations for finite-horizon ruin probabilities. ASTIN Bulletin 32(2): 267-281.

[11] Avanzi, B. 2009. Strategies for dividend distribution: A review. North American Actuarial Journal 13(2): 217-251.

[12] Avanzi, B., Cheung, E.C.K., Wong, B. and Woo, J.-K. 2013. On a periodic dividend barrier strategy in the dual model with continuous monitoring of solvency. Insurance: Mathematics and Economics 52(1): 98-113.

[13] Avanzi, B., Gerber, H.U. And Shiu, E.S.W. 2007. Optimal dividends in the dual model. Insurance: Mathematics and Economics 41(1): 111-123.

[14] Avanzi, B., Tu, V. And Wong, B. 2014. On optimal periodic dividend strategies in the dual model with diffusion. Insurance: Mathematics and Economics 55: 210-224.

[15] CARr, P. 1998. Randomization and the American put. Review of Financial Studies 11(3): 597-626.

[16] Chen, X., Xiao, T. And Yang, X. 2014. A Markov-modulated jump-diffusion risk model with randomized observation periods and threshold dividend strategy. Insurance: Mathematics and Economics 54: 76-83.

[17] DE FinetTi, B. 1957. Su un'impostazione alternativa della teoria collettiva del rischio. Transactions of the XVth International Congress of Actuaries 2: 433-443.

[18] Dickson, D.C.M. and Waters, H.R. 2004. Some optimal dividends problems. ASTIN Bulletin 34(1): 49-74.

[19] Gerber, H.U. 1979. An Introduction to Mathematical Risk Theory. Huebner Foundation Monograph 8. Homewood, IL: Richard D. Irwin. 
[20] Gerber, H.U., Lin, X.S. and Yang, H. 2006. A note on the dividends-penalty identity and the optimal dividend barrier. ASTIN Bulletin 36(2): 489-503.

[21] Gerber, H.U. And Shiu, E.S.W. 1998. On the time value of ruin. North American Actuarial Journal 2(1): 48-72.

[22] Gerber, H.U., Shiu, E.S.W. And Yang, H. 2012. The Omega model: From bankruptcy to occupation times in the red. European Actuarial Journal 2(2): 259-272.

[23] Kyprianou, A.E. And Pistorius, M.R. 2003. Perpetual options and Canadization through fluctuation theory. Annals of Applied Probability 13(3): 1077-1098.

[24] Lin, X.S., Willmot, G.E. And Drekic, S. 2003. The compound Poisson risk model with a constant dividend barrier: analysis of the Gerber-Shiu discounted penalty function. Insurance: Mathematics and Economics 33(3): 551-566.

[25] Loeffen, R.L. 2008. On optimality of the barrier strategy in de Finetti's dividend problem for spectrally negative Lévy processes. Annals of Applied Probability 18(5): 1669-1680.

[26] Ramaswami, V., Woolford, D.G. and Stanford, D.A. 2008. The Erlangization method for Markovian fluid flows. Annals of Operations Research 160(1): 215-225.

[27] Stanford, D.A., Avram, F., Badescu, A.L., Breuer, L., Da Silva Soares, A. And Latouche, G. 2005. Phase-type approximations to finite-time ruin probabilities in the SparreAnderson and stationary renewal risk models. ASTIN Bulletin 35(1): 131-144.

[28] Stanford, D.A., Yu, K. And Ren, J. 2011. Erlangian approximation to finite time ruin probabilities in perturbed risk models. Scandinavian Actuarial Journal 2011(1): 38-58.

[29] Zhang, Z. 2014. On a risk model with randomized dividend-decision times. Journal of Industrial and Management Optimization 10(4): 1041-1058.

[30] Zhang, Z. And Cheung, E.C.K. 2014a. The Markov additive risk process under an Erlangized dividend barrier strategy. Methodology and Computing in Applied Probability. In press.

[31] Zhang, Z. And Cheung, E.C.K. 2014b. A note on a Lévy insurance risk model under periodic dividend decisions. Preprint. 